\title{
Dynamics of the Transition from Spiral Rainbands to a Secondary Eyewall in Hurricane Earl (2010)
}

\author{
ANTHONY C. DiDLAKE JR. \\ Department of Meteorology and Atmospheric Science, The Pennsylvania State University, University Park, Pennsylvania \\ PAUl D. REASOR AND ROBERT F. ROGERS \\ NOAA/Atlantic Oceanographic and Meteorological Laboratory/Hurricane Research Division, Miami, Florida \\ Wen-CHAU LeE \\ National Center for Atmospheric Research, Boulder, Colorado
}

(Manuscript received 20 November 2017, in final form 24 May 2018)

\begin{abstract}
Airborne Doppler radar captured the inner core of Hurricane Earl during the early stages of secondary eyewall formation (SEF), providing needed insight into the SEF dynamics. An organized rainband complex outside of the primary eyewall transitioned into an axisymmetric secondary eyewall containing a low-level tangential wind maximum. During this transition, the downshear-left quadrant of the storm exhibited several notable features. A mesoscale descending inflow (MDI) jet persistently occurred across broad stretches of stratiform precipitation in a pattern similar to previous studies. This negatively buoyant jet traveled radially inward and descended into the boundary layer. Farther inward, enhanced low-level inflow and intense updrafts appeared. The updraft adjacent to the MDI was likely triggered by a region of convergence and upward acceleration (induced by the negatively buoyant MDI) entering the high $-\theta_{\mathrm{e}}$ boundary layer. This updraft and the MDI in the downshear-left quadrant accelerated the tangential winds in a radial range where the axisymmetric wind maximum of the secondary eyewall soon developed. This same quadrant eventually exhibited the strongest overturning circulation and wind maximum of the forming secondary eyewall. Given these features occurring in succession in the downshear-left quadrant, we hypothesize that the MDI plays a significant dynamical role in SEF. The MDI within a mature rainband complex persistently perturbs the boundary layer, which locally forces enhanced convection and tangential winds. These perturbations provide steady low-level forcing that projects strongly onto the axisymmetric field, and forges the way for secondary eyewall development via one of several SEF theories that invoke axisymmetric dynamical processes.
\end{abstract}

\section{Introduction}

Secondary eyewalls are frequently observed phenomena in intense tropical cyclones (TCs). Many studies show that these features form when convection outside of a preexisting primary eyewall coalesces into a connected ring with a collocated axisymmetric tangential wind maximum (e.g., Willoughby et al. 1982; Black and Willoughby 1992; Dodge et al. 1999; Houze et al. 2007; Didlake and Houze 2011; Bell et al. 2012). Once a secondary eyewall forms, characteristic changes in size and intensity usually occur, which makes predicting these

\footnotetext{
Corresponding author: Anthony C. Didlake Jr., didlake@ psu.edu
}

inner-core features a high priority for forecasters (Sitkowski et al. 2011, 2012; Kossin and Sitkowski 2012; Yang et al. 2013; Kossin and DeMaria 2016; Zhang et al. 2017). Despite their frequent occurrence, secondary eyewalls remain a forecasting challenge in part because the underlying mechanisms for secondary eyewall formation are not completely understood.

There has been a variety of physical processes proposed to explain secondary eyewall formation (SEF). One process involves vortex Rossby waves propagating within a storm's inner core away from the primary eyewall, which then stagnate and build an axisymmetric secondary wind maximum (Montgomery and Kallenbach 1997). Many studies have found evidence of these propagating waves in both models and observations 
(e.g., Corbosiero et al. 2006; Hogsett and Zhang 2009; Martinez et al. 2011; Abarca and Corbosiero 2011). However, current understanding points to vortex Rossby waves as having a more supportive role in SEF (Qiu et al. 2010) or no role at all (Judt and Chen 2010), while the primary contributing process entails the axisymmetrization of rainband convection originating from outer regions. Judt and Chen (2010) argued that rainband convection acts as local sources of diabatic heating, which, when sufficiently projected onto the axisymmetric mean, produces a local potential vorticity maximum that leads to secondary eyewall development. Terwey and Montgomery (2008) proposed that secondary eyewalls form when sporadic rainband convection occurs along a negative radial gradient of axisymmetric vorticity, where the resulting potential vorticity anomalies then cascade upscale to form a secondary potential vorticity maximum. Other studies have focused on boundary layer mechanisms for spinning up an axisymmetric secondary wind maximum. Wu et al. (2012), Huang et al. (2012), and Abarca and Montgomery (2013, 2014) found that unbalanced dynamics, appearing as supergradient boundary layer flow, largely governs the axisymmetric development of a secondary eyewall following a broadening of the vortex wind field. Alternatively, Kepert (2013) concluded that axisymmetric balanced dynamics can spin up a secondary wind maximum through a feedback between frictional convergence, convection, and radial vorticity. Therein, the boundary layer evolution and its unbalanced flow only occur as a response to the wind and pressure field at the top of the boundary layer. Sun et al. (2013) found that both unbalanced and balanced dynamics contribute significantly to developing a secondary eyewall.

Given that a sufficient amount of rainband convection is necessary for each proposed SEF mechanism, it is important to understand the controls on rainband activity. Environmental moisture has commonly been claimed as a key factor (e.g., Kimball 2006). Hill and Lackmann (2009) showed that simulated TCs initialized in moisture environments at all altitudes produced more widespread and persistent rainband convection. Alternatively, Xu and Wang (2010) found that surface entropy fluxes in the outer regions were the critical factor for rainband production, where larger fluxes produced greater CAPE and more convection.

Substantial latent heating from increased rainband activity acts to spin up the tangential winds of the outer core, thereby radially expanding the wind field of the storm (Maclay et al. 2008; Smith et al. 2009). This storm expansion is a process that commonly precedes SEF in both observations and models (Bell et al. 2012; Huang et al. 2012). Rozoff et al. (2012) examined the roles of outer-core latent heating and storm expansion in SEF. They determined that increasing outer-core winds improves the axisymmetric efficiency of converting heat energy released by rainband convection into increased kinetic energy, which can be exhibited locally as a developing secondary wind maximum. It remains unclear whether the expanding wind field is a causal process for SEF or a consequence of SEF mechanisms already initiated by increased rainband convection.

In the context of explaining SEF, most previous studies approach rainband convection as sporadic features that assert their influence on the evolving vortex structure only through their vortex-scale axisymmetric projection. But TC rainbands are asymmetric structures that have their own intricate organization on the convective scale and the mesoscale. The organization occurring on these smaller scales may exhibit certain characteristics that initiate the upscale development of a secondary eyewall. Therefore, in order to fully understand the earliest mechanisms that trigger SEF, we need to better understand the asymmetric rainband patterns that lead to a prominent axisymmetric projection and wind field expansion, both of which mark the pathway for most hypothesized SEF mechanisms.

TC rainbands tend to form an asymmetric complex when the storm experiences environmental vertical wind shear (Willoughby et al. 1984; Hence and Houze 2012b). In this complex, convective precipitation concentrates in the right-of-shear half, where enhanced lowlevel vorticity and moisture tend to coincide (Riemer 2016). Here, isolated or connected convective cells are initiated and form the upwind end of the larger spiral rainband complex (Didlake and Houze 2013a). In the left-of-shear half, stratiform precipitation is predominant in the downwind end of the complex (May and Holland 1999; Didlake and Houze 2013b). The rainband features associated with this downwind region project more strongly onto the axisymmetric structure given both the mesoscale, homogeneous nature of stratiform precipitation and the fact that these features occur at a smaller radius. Thus, it seems that the downwind end of the rainband complex may play an important role in the early stages of SEF.

Recent modeling studies have pointed to stratiform processes occurring in the axisymmetric average as the catalyst for both the expansion of the wind field and the development of a low-level tangential wind maximum. This approach is a "top-down" pathway to SEF. Robust latent heating starts in the middle levels, associated with vorticity and updraft anomalies. These features then descend (presumably by downward advection) into the low levels where a deep overturning circulation subsequently develops and leads to the secondary eyewall (Fang and Zhang 2012; Zhang et al. 2017). These studies show that stratiform processes within TC rainbands are 
projecting strongly enough onto the axisymmetric average to form a secondary eyewall; however, the smaller-scale, asymmetric details of the responsible rainband features still needed to be thoroughly examined.

In an observational study of Hurricane Rita, Didlake and Houze (2013b) found that the downwind stratiform region of the rainband complex exhibited a mesoscale descending inflow jet similar to the rear-to-front jet of leading-line mesoscale convective systems (MCSs; Houze 2004). At the innermost point of the inflow jet, air suddenly plummets from the middle levels into the boundary layer, which then connects to a local enhancement of the boundary layer radial inflow. Considering that this inflow surge and related vorticity enhancement occurred over a significant portion of the storm, it likely had an impact on the larger vortex circulation. Rita developed a secondary eyewall hours later (Didlake and Houze 2011; Bell et al. 2012), but no direct connection between the two features was established. In their modeling study, Qiu and Tan (2013) found that a similar descending inflow in the stratiform rainband region reached the boundary layer and locally enhanced the low-level inflow, albeit not in the sudden fashion as observed in Rita. They connected the asymmetric enhanced boundary layer flow to unbalanced axisymmetric dynamics that led to SEF in the processes described by Huang et al. (2012) and Abarca and Montgomery (2013).

In this study, we further explore the possible connection between stratiform rainband processes and SEF by examining observations of Hurricane Earl (2010). Earl was intensely sampled by multiple aircraft in conjunction with three field campaigns and U.S. Air Force reconnaissance. Previous studies have explored several aspects of Earl's evolution with these observations, including focus on rapid intensification processes, eyewall asymmetries, environmental interaction, low-level structure, and warm core structure (Montgomery et al. 2014; Stevenson et al. 2014; Rogers et al. 2015; Susca-Lopata et al. 2015; Stern and Zhang 2016). We will examine a combination of airborne radar, land-based radar, and flight-level observations to analyze the structural and dynamical evolution of Earl's rainbands into the secondary eyewall that formed after its rapid intensification stage.

\section{Data and methodology}

The airborne radar data utilized in this study were collected by the NOAA WP-3D (P3) Tail Doppler radar (TDR), which is an X-band Doppler radar that scans around a horizontal axis in cones pointing $\sim 20^{\circ}$ fore and aft (Jorgensen and DuGranrut 1991). With this scanning technique, two independent looks of the three-dimensional wind vector are collected as the plane flies along its track. The data used in this study come from four P3 missions on 29 August 2010 and 30 August 2010: 20100829H1, 20100829I1, 20100830H1, and $20100830 \mathrm{I} 1$.

The P3 TDR data first undergo an automated quality control and dealiasing procedure that produces errors comparable to those from manual processing (Rogers et al. 2012). Next, the three-dimensional wind fields are retrieved using a variational technique (Gamache 1997; Gao et al. 1999; Reasor et al. 2009) that optimizes the radar projection equation constrained by mass continuity and boundary conditions. These wind fields along with the three-dimensional reflectivity field were solved onto a Cartesian grid with horizontal and vertical resolution of 2 and $0.5 \mathrm{~km}$, respectively. The storm motion averaged over each mission was removed from the winds. Storm centers were objectively determined using a real-time analysis of flight level data (Willoughby and Chelmow 1982). The data were then interpolated to storm-centered cylindrical coordinates with a radial, vertical, and azimuthal resolution of $2 \mathrm{~km}, 0.5 \mathrm{~km}$, and $0.5^{\circ}$, respectively. Also on board the NOAA P3 was a C-band lower-fuselage (LF) radar that scans around a vertical axis, continually observing storm reflectivity over a larger scale. We analyze these data with respect to the 200-850-hPa deeplayer environmental vertical wind shear, which is taken from the Statistical Hurricane Intensity Prediction Scheme (SHIPS) database (DeMaria et al. 2005).

Additional airborne data come from flight-level observations. The FLIGHT+ dataset (Vigh et al. 2016) is a collection of flight-level data from NOAA P3 and U.S. Air Force (USAF) C-130 reconnaissance aircraft that are segmented into radial legs relative to the storm center. The current study utilizes this dataset, with focus on the $700 \mathrm{hPa}-$ level observations.

Land-based radar observations were collected by the U.S. National Weather Service WSR-88D at San Juan, Puerto Rico (TJUA). This S-band radar operates with a scanning volume at fixed elevation angles $\left(0.5^{\circ}-19.5^{\circ}\right)$ that lasts $5-12 \mathrm{~min}$. The current study uses observations of Hurricane Earl collected during the time span of 1700 UTC 30 August 2010-0600 UTC 31 August 2010. Axisymmetric tangential winds of Earl were retrieved by applying the ground-based velocity track display (GBVTD; Lee et al. 1999) technique, providing averaged velocities at each radius over time.

\section{Overview of Earl's secondary eyewall formation in an axisymmetric framework}

Hurricane Earl began as a westward-tracking Cape Verde tropical wave that became a tropical storm on 
a) $08 / 29 / 101102 \mathrm{Z}$

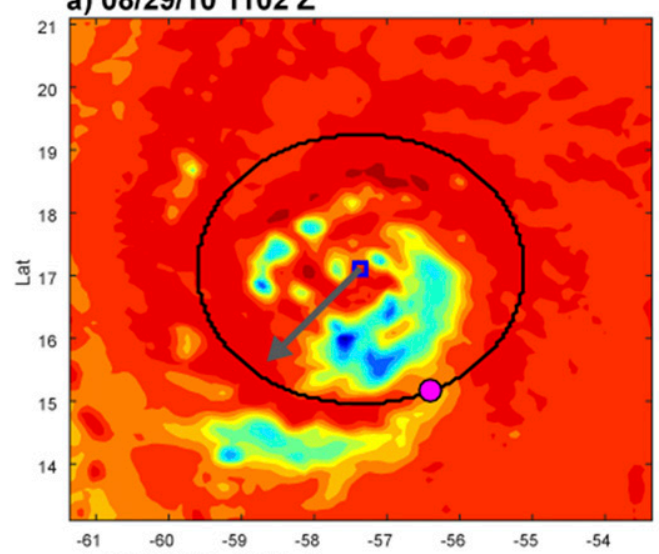

c) $08 / 30 / 101226 Z$

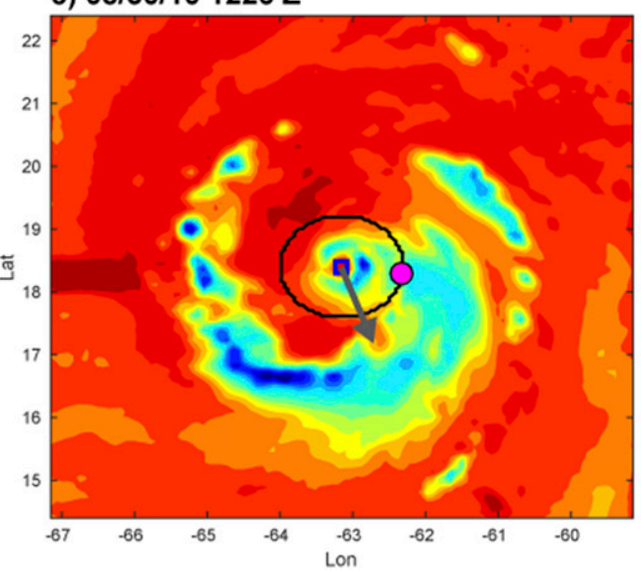

b) $08 / 29 / 102351 \mathrm{Z}$

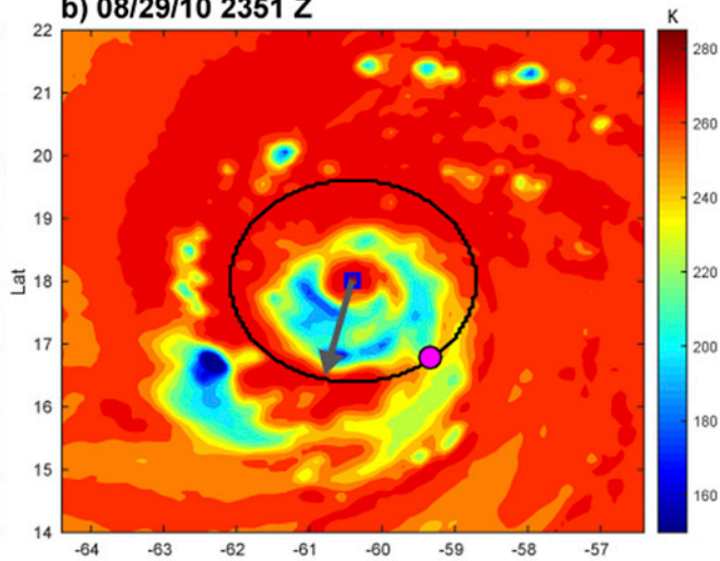

d) $08 / 31 / 100438 \mathrm{Z}$

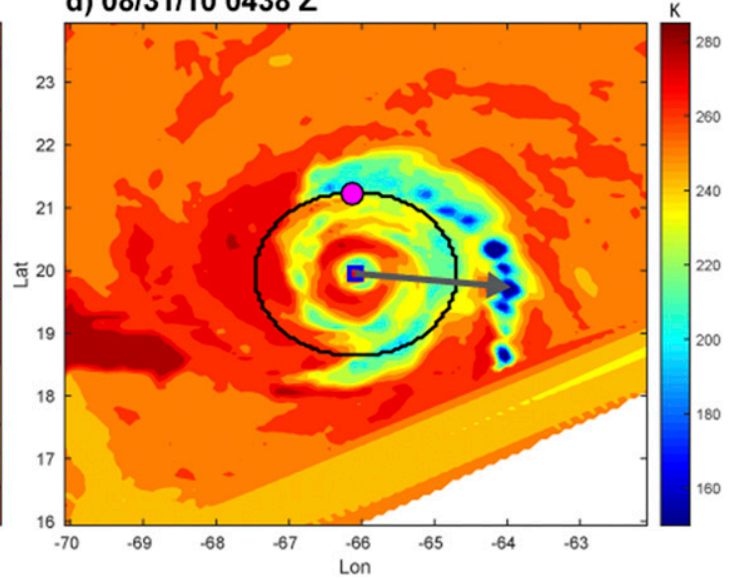

FIG. 1. The 85-91-GHz brightness temperatures of Hurricane Earl from (a)-(c) the Special Sensor Microwave Imager/Sounder and (d) the TRMM Microwave Imager at the four noted times. For each image, the gray arrow is the 200-850-hPa environmental wind shear vector pointing from the storm center located at the blue square. The black ring and the pink circle mark the radius and azimuth where outer rainband convection connects to inner-core convection. See text for details.

26 August 2010. As Earl approached the Lesser Antilles on 29 August, it rapidly intensified from a $28 \mathrm{~m} \mathrm{~s}^{-1}$ tropical storm to a $59 \mathrm{~m} \mathrm{~s}^{-1}$ category- 3 hurricane over the next $36 \mathrm{~h}$. More details on Earl's life cycle can be found in Cangialosi (2010). The data analyzed in the current study focus on rainband convection that occurs after the onset of rapid intensification.

Figure 1 presents satellite microwave $(85-91 \mathrm{GHz})$ imagery of Earl after the onset of rapid intensification and leading up to SEF. In Figs. 1a-c, rainband convection is organized into a typical stationary band complex (SBC; Willoughby et al. 1984), where the spiral complex remains relatively steady in relation to the deep-layer environmental wind shear vector. Also in Fig. 1, an estimated connecting point between the SBC and inner-core convection, which marks the downwind end of the SBC, is shown by the pink dot. This location is determined first by identifying the radius of a local maximum in the azimuthally averaged brightness temperatures, then by locating the azimuth of a local brightness temperature minimum. As the shear vector shifts counterclockwise over time, this connecting point also shifts azimuthally in a similar

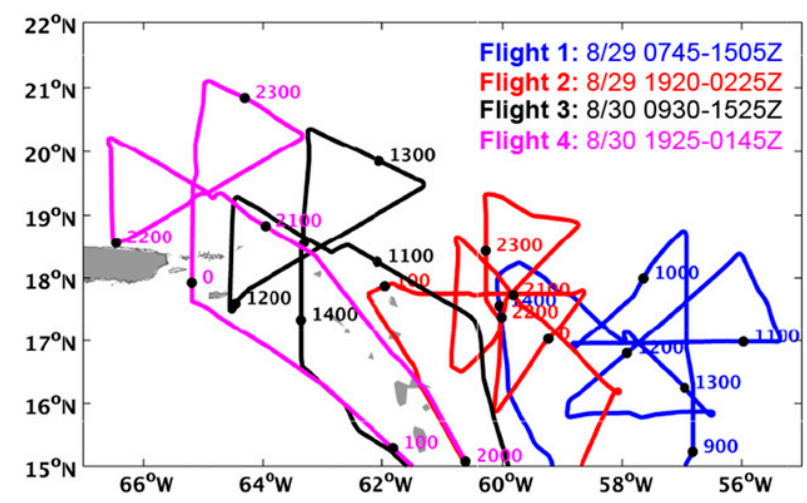

FIG. 2. Flight tracks of four NOAA P3 missions into Hurricane Earl. Hour markers (UTC) are shown on each flight track. 


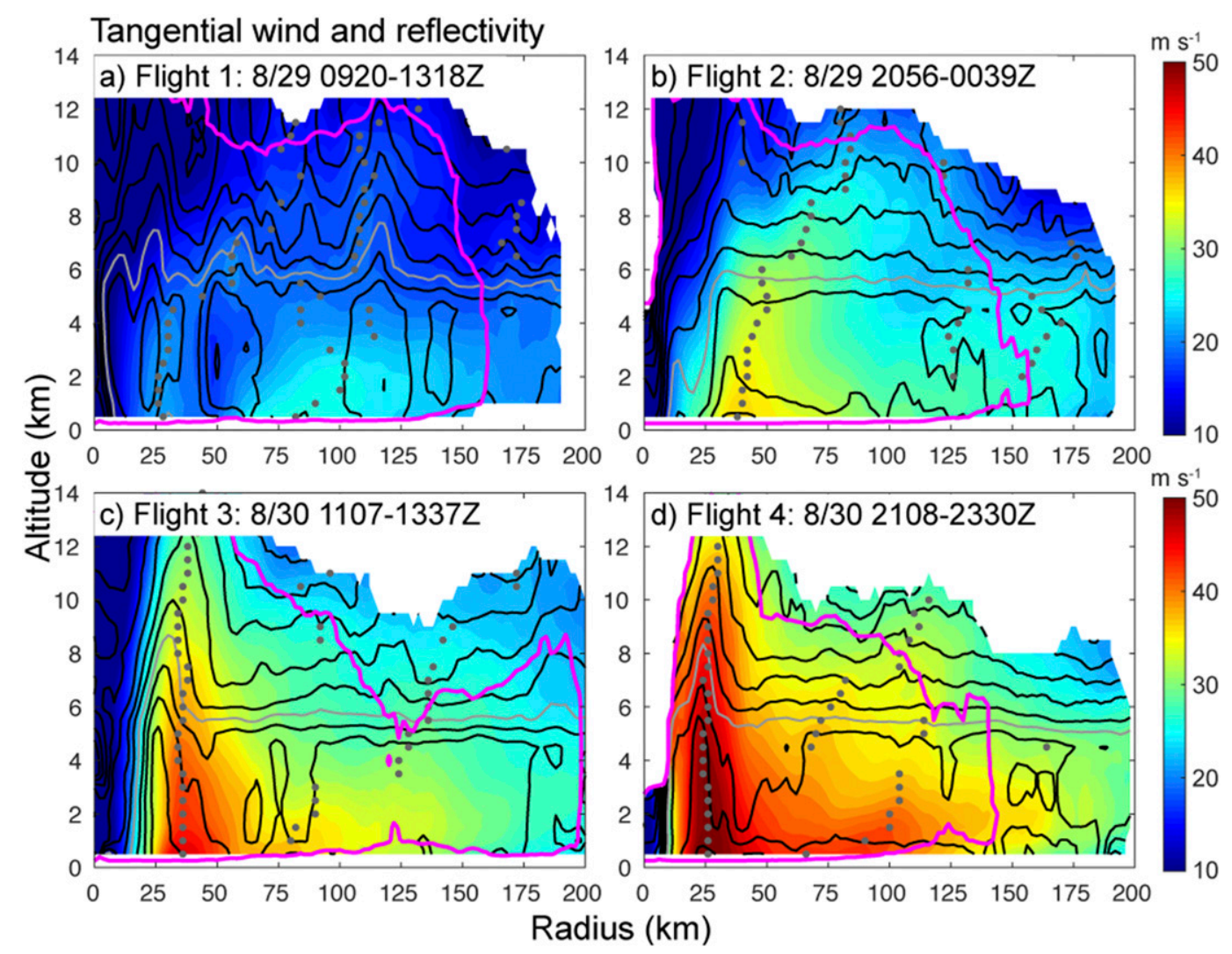

FIG. 3. Azimuthal average of tangential velocity (color shading) and reflectivity (black contours) from the TDR observations at flights 1-4 during the times indicated. The pink line outlines locations with $>50 \%$ azimuthal coverage of available data. Local maxima of tangential wind are marked as gray dots. Reflectivity contours are at 5-dBZ intervals, with the gray contour being the $25-\mathrm{dB} Z$ contour for flights 1 and 3 and the $30-\mathrm{dB} Z$ contour for flights 2 and 4 .

fashion, and thus remains left of the shear vector. This shear-relative orientation of the SBC is consistent with several other observational and modeling studies (e.g., Hence and Houze 2012b; Didlake and Houze 2013a,b; Riemer 2016). The rainband convection also appears to increase over time. By 31 August (Fig. 1d), the same spiral rainband complex is less prominent, but rather there is a clear circular ring of convection around the eyewall, suggesting the development of a secondary eyewall.

Figure 2 shows the flight tracks for the four NOAA P3 missions used in this study. Figure 3 shows the TDR axisymmetric tangential winds from these flights, which cover a similar time span as in Fig. 1. Local maxima in tangential winds were determined using an algorithm that considers the width and separation distances of the peaks. ${ }^{1}$

\footnotetext{
${ }^{1}$ The specific algorithm used was "findpeaks" in MATLAB using a minimum peak width of $8 \mathrm{~km}$ and peak separation distance of $24 \mathrm{~km}$ (http://www.mathworks.com/help/signal/ref/ findpeaks.html).
}

These maxima are marked as dots at each altitude. The wind field began in Fig. 3a with two tangential wind maxima in the low levels. During this phase as a tropical storm, the strongest winds were found in the outer maximum associated with an outer rainband. In Fig. 3b, the eyewall appeared and contained the overall wind maximum. Over time, this wind maximum strengthened rapidly and decreased in radius. Simultaneously outside of the eyewall, the tangential winds increased at every radius throughout all altitudes, indicating the expansion of the wind field. This wind field expansion coincided with the observed increase in rainband convection, as rainband heating is expected to spin up the storm's outer circulation (Smith et al. 2009; Moon and Nolan 2010).

In flight 2, two local maxima occurred near 125and $155-\mathrm{km}$ radius in the low to midlevels, both radially outside the primary eyewall. In flight 3 , two local maxima now occur near $80-90$ and $125-135 \mathrm{~km}$. The inner maximum here mostly remained below $4 \mathrm{~km}$, 
Radial and vertical velocity
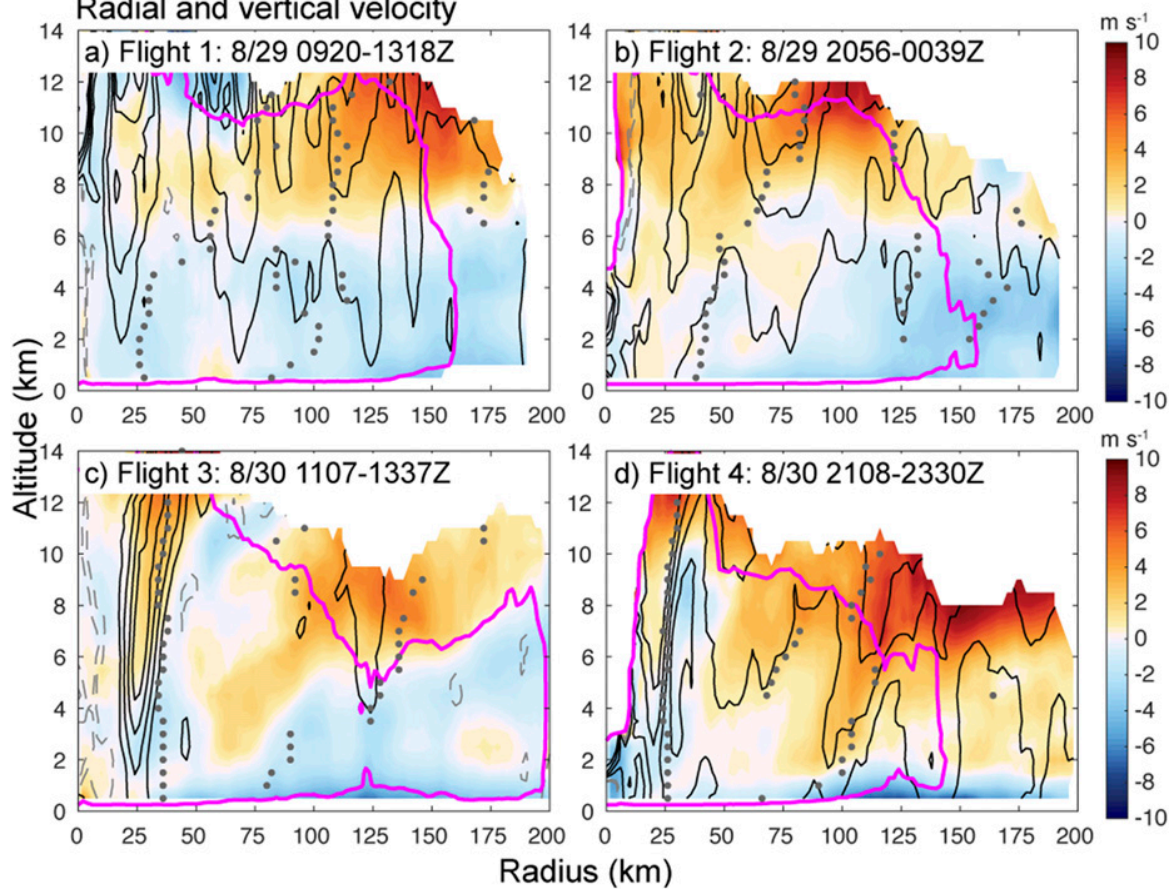

FIG. 4. Azimuthal average of radial velocity (color shading) and vertical velocity (black and gray contours) from the TDR observations for flights 1-4. Updrafts (black) are contoured at $0.4,0.8,1.2$, and $1.6 \mathrm{~m} \mathrm{~s}^{-1}$, and downdrafts (gray) are contoured at -0.2 and $-0.4 \mathrm{~m} \mathrm{~s}^{-1}$. The pink line outlines locations with $>50 \%$ azimuthal coverage of available data. Local maxima of tangential wind are marked as gray dots.

while the outer maximum stretched from 4-km altitude and upward. By flight 4 , two clear maxima again occurred with pronounced outward slopes. The inner maximum near $60 \mathrm{~km}$ is most prominent above $4 \mathrm{~km}$, while the outer maximum between 80 and $120 \mathrm{~km}$ again appeared between 1 - and $\sim 6$-km altitude.

Figure 4 shows the azimuthally averaged radial and vertical velocities corresponding to Fig. 3. In flights $1-3$, the
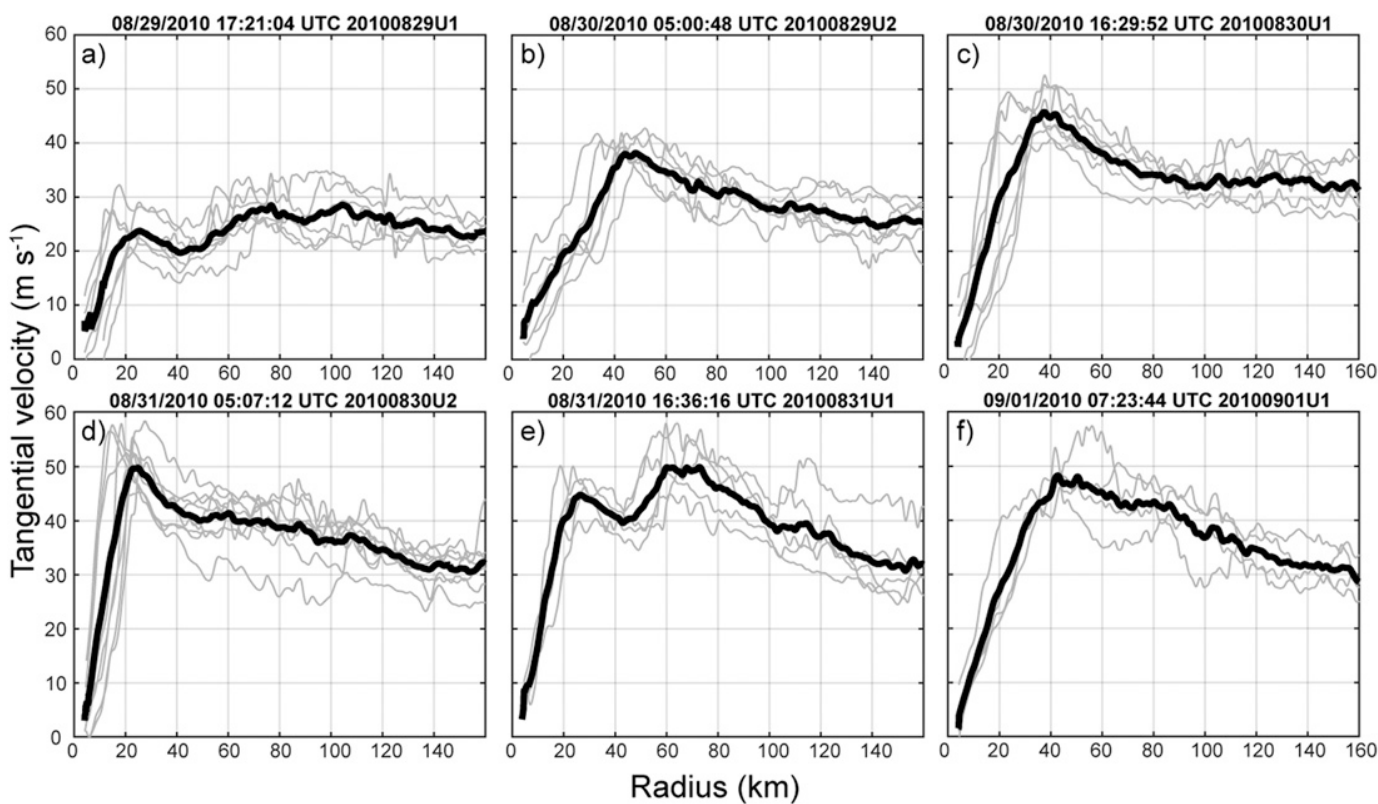

FIG. 5. Flight-level tangential velocity for the radial legs (gray lines) and the azimuthal average (black line) from the USAF flight missions indicated. Flight levels are at (a) $850 \mathrm{hPa}$ and (b)-(f) $700 \mathrm{hPa}$. 
a) Flight 2: 08/29 $2038 Z$

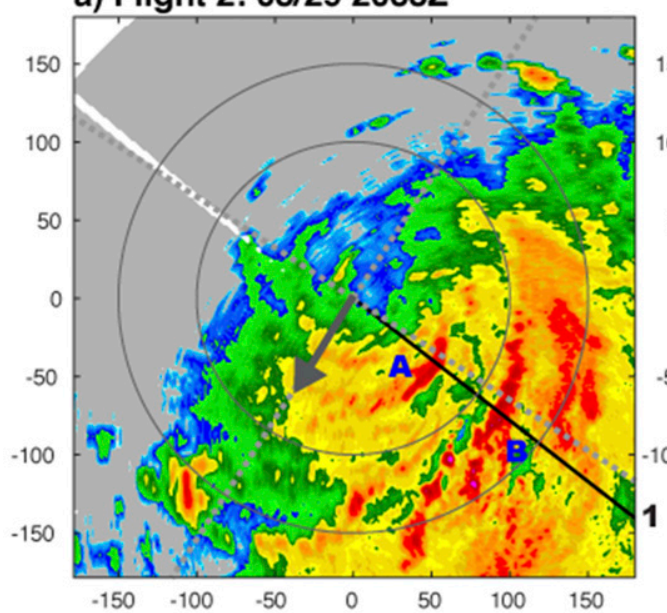

c) Flight 3: 08/30 $1053 \mathrm{Z}$

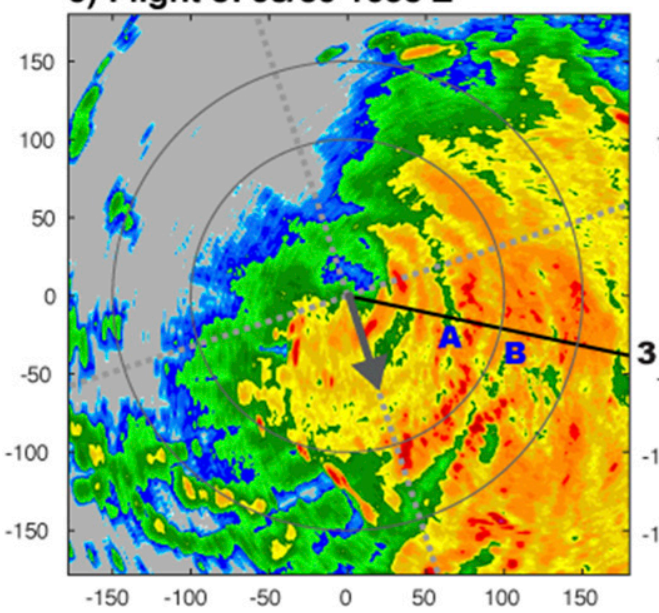

b) Flight 2: 08/29 $2347 Z$

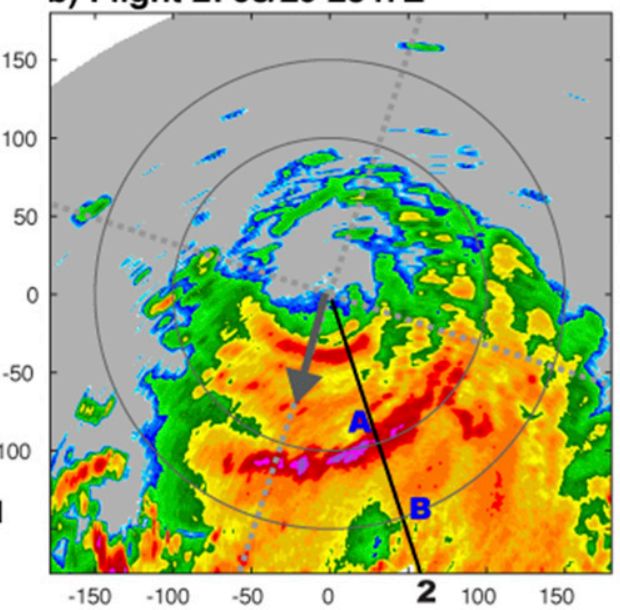

d) Flight 4:08/30 $2226 \mathrm{Z}$

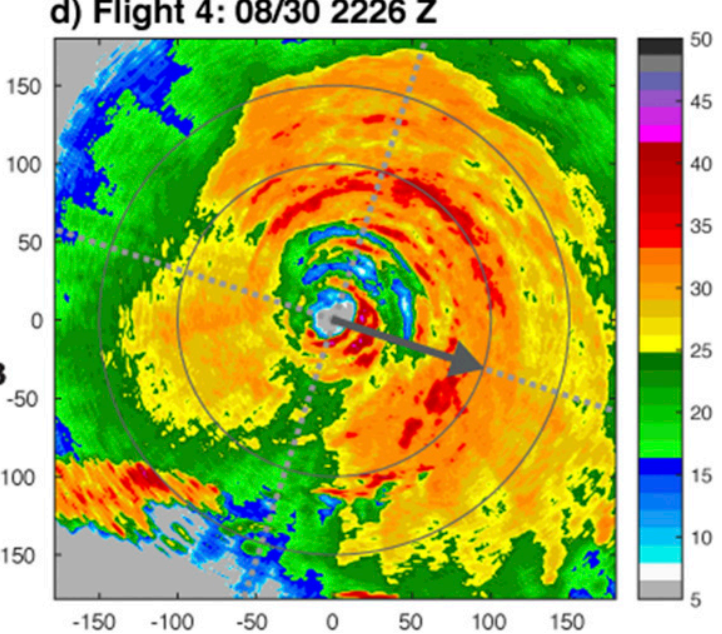

$\mathrm{dBZ}$

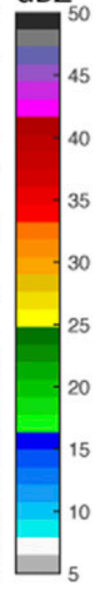

FIG. 6. The LF radar reflectivity at specified times. For each time, the 200-850-hPa environmental wind shear vector (gray) points from the storm center. Black lines are cross sections (slices 1-3) shown in Figs. 7, 8, and 10, respectively. The labels $\mathrm{A}$ and $\mathrm{B}$ mark the updraft features in the same cross-section figures.

dominant radial circulation is deep inflow extending from the lowest level to $6 \mathrm{~km}$ and outflow above. The updraft and outflow of the primary eyewall strengthens over this time of rapid intensification. Outside of the primary eyewall at flight 3, a clear region of radial outflow begins near 2-km altitude and 60-km radius and extends to higher altitudes. Further inspection of this feature indicates an axisymmetric updraft of $0.25 \mathrm{~m} \mathrm{~s}^{-1}$. This channel of air is associated with the outer tangential maximum near $85-\mathrm{km}$ radius (cf. Fig. 3c). Rising motion is also collocated with the outer wind maximum near $125 \mathrm{~km}$. During flight 4, there are two rising outflow channels (50-60 and 80-100 km) that are each associated with the two local tangential wind maxima shown in Fig. 3d, matching in both radii and altitudes.

The NOAA P3s did not fly on the next day (31 August), which was also when satellite observations displayed an outer ring of convection (Fig. 1d). But the USAF C-130 fleet continued their twice-daily surveillance missions, capturing the tangential wind evolution during secondary eyewall development. Figure 5 presents radial profiles of the flight-level tangential wind during this time. These data confirm Earl's initial double wind maximum, tangential wind intensification, and the contraction of the radius of maximum wind (Figs. 5a-d). A bulge in the outer wind field $(\sim 60-100 \mathrm{~km})$ is present in Fig. 5d, which matches the time of the observed ring of convection (cf. Fig. 1d). The secondary eyewall was clearly forming in this region. At $13 \mathrm{~h}$ later in Fig. 5e, the outer wind maximum between $60-$ and $75-\mathrm{km}$ radius had matured and surpassed the inner eyewall wind maximum. In Fig. 5f, the eyewall replacement cycle was complete, as the inner eyewall had dissipated. The evolution of the flight level tangential winds 
Flight 2: $2038 Z$
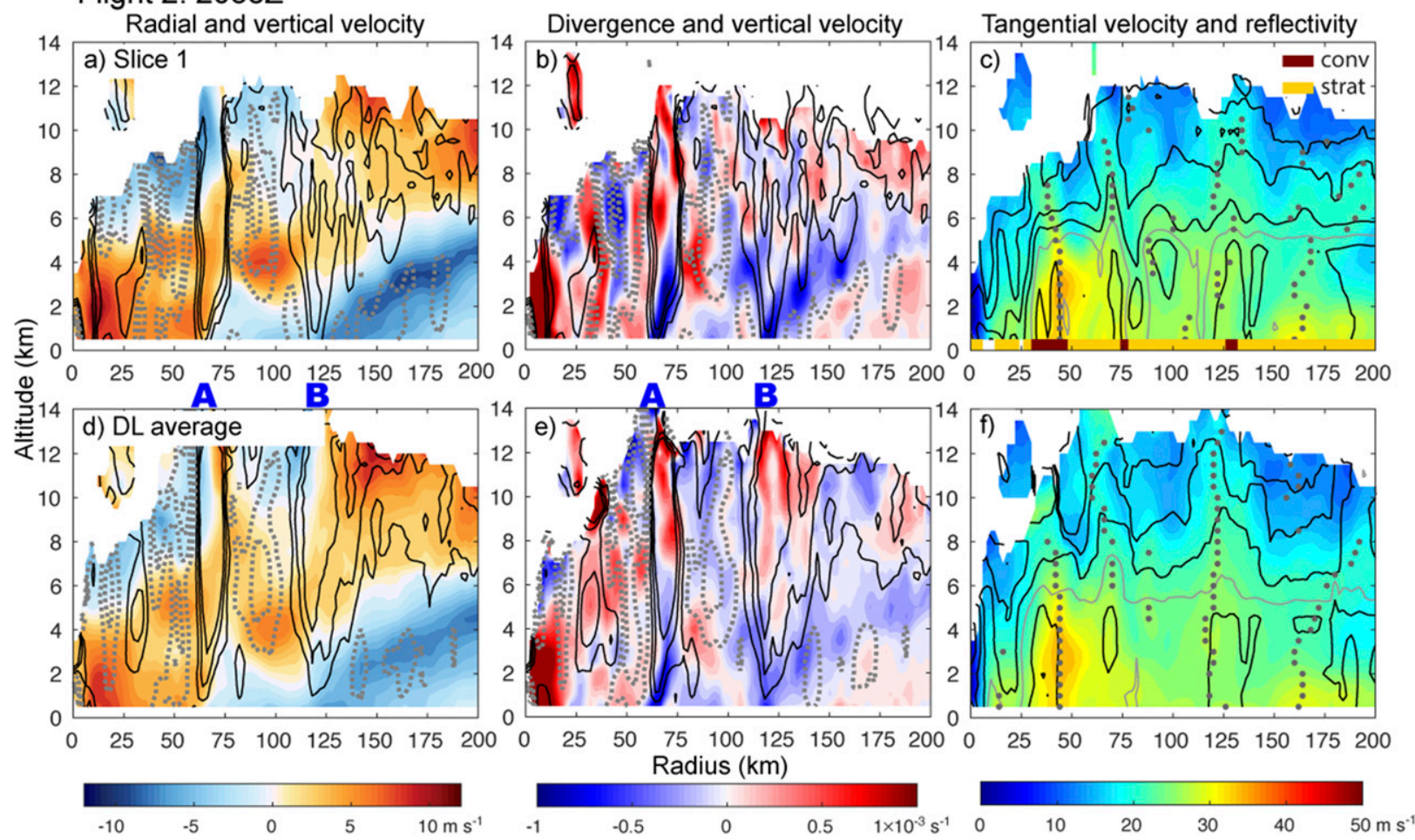

FIG. 7. (a) Cross section of radial (color shading) and vertical velocity (contours) at slice 1 of flight 2 at 2038 UTC as shown in Fig. 6a. Updrafts (black) are contoured at $0.5,1$, and $1.5 \mathrm{~m} \mathrm{~s}^{-1}$, and downdrafts (gray) are contoured at $-0.1,-0.5$, and $-1 \mathrm{~m} \mathrm{~s}^{-1}$. The letters A and B mark updraft features as in Fig. 6a and discussed in the text. (b) Divergence (color shading) with vertical velocity contours. (c) Tangential velocity (color shading) with local tangential velocity maxima marked as gray dots; reflectivity contours are overlaid at 5 -dBZ intervals, with the $25-\mathrm{dB} Z$ contour in gray. The lowest level of burgundy and orange shows locations of convective and stratiform precipitation, respectively. (d)-(f) As in (a)-(c), but for averaged cross-section fields of the downshear-left (DL) quadrant in Fig. 6a.

is consistent with the eyewall replacement study of Sitkowski et al. (2011).

The outer radii in Figs. 3 and 4 show signs of a developing secondary eyewall, having local tangential wind maxima coinciding with deep overturning circulations. However, by the time of the last P3 flight examined here (flight 4 ; cf. Figs. $3 \mathrm{~d}$ and $4 \mathrm{~d}$ ), there were two radial locations at which this combination was coherently occurring: $50-70$ and $80-100 \mathrm{~km}$. Shapiro and Willoughby (1982) demonstrated that an eyewall-like heat source in an axisymmetric vortex yields a maximum in low-level tangential wind tendency radially inward of the heat source, which implies that the wind maximum contracts over time. These same dynamics help explain the contraction of a developing secondary eyewall; specifically, such contraction of either tangential wind maximum (Fig. 3d) likely occurred in Earl, which would suggest that the outermost local maximum led to the secondary wind maximum between $60-$ and $75-\mathrm{km}$ radius in Fig. 5e.

In the next section, we examine Earl's asymmetric rainband features to determine which structures are most responsible for the axisymmetric tangential wind local maxima in flights $2-4$. We analyze these features chronologically in an effort to identify the earliest signs of a developing secondary eyewall circulation.

\section{Asymmetric structure and dynamics leading up to SEF}

Characterizing the asymmetric structure of rainband precipitation leading up to SEF is an important step to understanding how the precipitation features axisymmetrize into a concentric ring with an associated secondary low-level tangential wind maximum. In Figs. $4 \mathrm{~b}$ and $4 \mathrm{c}$, the azimuthal average of radial flow showed an inflow peak in the middle levels of the rainband region between 3 - and $6-\mathrm{km}$ altitude. The subsequent analysis of the asymmetric structure will show that this feature arose mostly from wind patterns in the downshear-left (DL) quadrant. This is the same quadrant as the downwind end of the strengthening rainband complex (Fig. 1), which is where stratiform precipitation tends to be predominant (Hence and Houze 2012b; Didlake and Houze 2013b). 
Flight 2: $2347 Z$

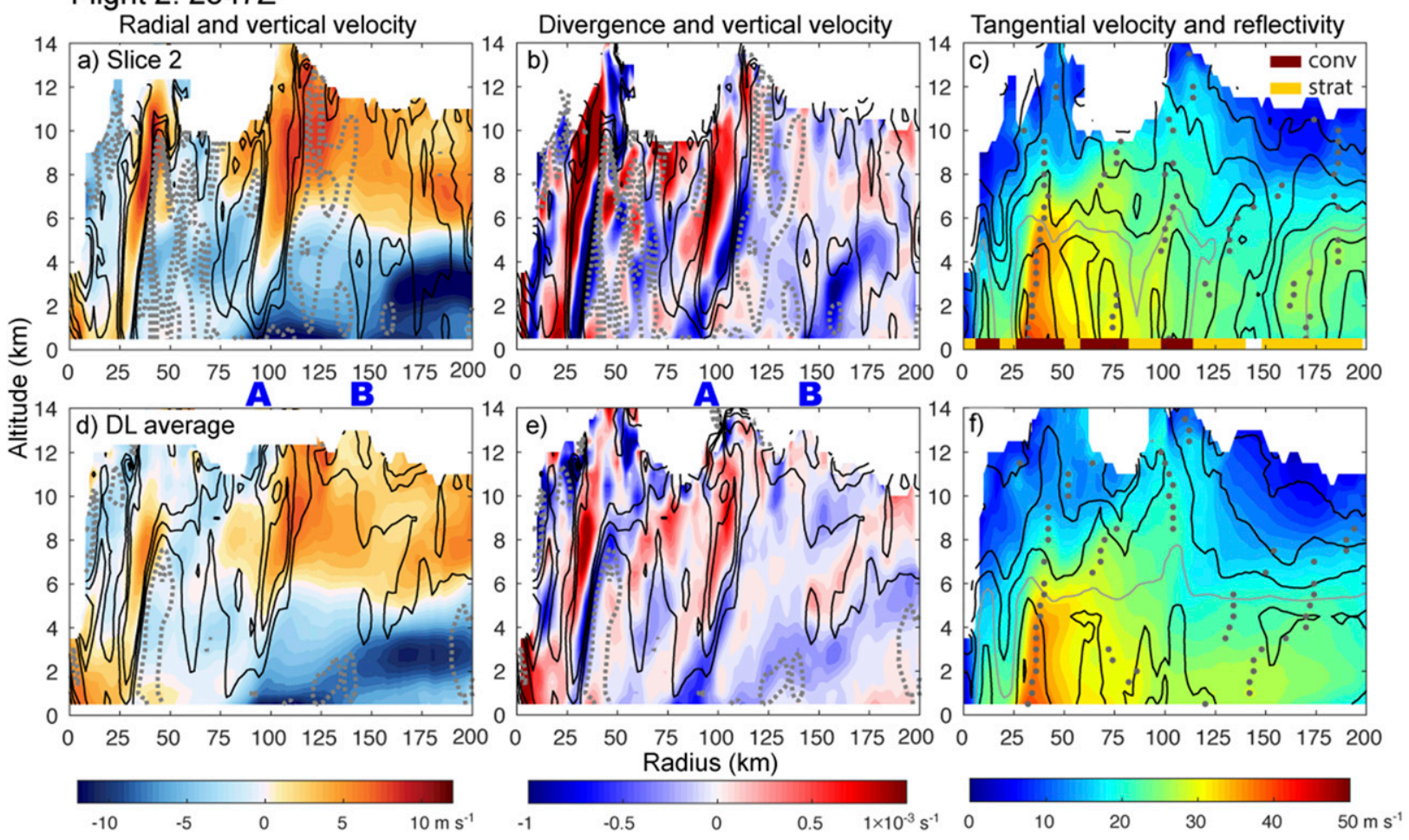

FIG. 8. As in Fig. 7, but for slice 2 and the DL average of flight 2 at 2347 UTC as shown in Fig. 6 b.

We now present a close examination of the DL quadrant observed by the P3 TDR.

\section{a. Flights 2 and 3}

Figure 6 shows single sweeps of LF radar data from each of the flights during flights $2-4$. These images were taken at times when the radar signal best captured the DL quadrant precipitation without attenuation from the eyewall. At flights 2 and 3, the DL quadrant appears to have broad patches of moderate reflectivity and discrete or connected bands of more intense reflectivity in the rainband region. This signal suggests mixed stratiform and convective precipitation with no clear dominance of either precipitation regime. The apparent stratiform patches occur radially outward of the convective cells.

Figure 7 presents TDR data through a DL cross section (slice 1) shown in Fig. 6a, as well as the azimuthally averaged cross section of all available data in the DL quadrant at this time. The convective-stratiform separation technique used in Didlake and Houze (2009) is applied to the reflectivity field to determine the precipitation regime that is dominant at each radial location. In slice 1 , stratiform precipitation is present outside of $130-\mathrm{km}$ radius (Fig. $7 \mathrm{c}$ ). Within this stratiform precipitation, radial inflow is organized into a midlevel tongue that is collocated with downdrafts. The maximum inflow occurs at 4-km altitude, which is just below the reflectivity brightband at $4.5-\mathrm{km}$ altitude. The inflow then descends and reaches the boundary layer around $130-\mathrm{km}$ radius. The tangential winds here (Fig. 7c) are locally enhanced beneath the inflow jet in the lower levels around $160 \mathrm{~km}$. Figure $7 \mathrm{~b}$ shows a continuous region of convergence along the inner and upper sides of the inflow jet. Updrafts sprout from local maxima of midlevel convergence near 135and $155-\mathrm{km}$ radius. But the strongest and deepest updraft, marked B, originates in the boundary layer and occurs at the point where the inflow jet (and associated convergence) reaches the boundary layer. This collocation suggests that updraft $B$ is dynamically connected to the descending inflow jet. Updraft $B$ is also associated with a tangential wind maximum at $3-5-\mathrm{km}$ altitude. Radially inward, boundary layer inflow continues until reaching another strong updraft near $70-\mathrm{km}$ radius, marked $\mathrm{A}$. Updrafts $\mathrm{A}$ and $\mathrm{B}$ correspond to the two convective lines labeled in Fig. 6a (note that the eyewall located at 40-km radius has a weaker updraft in these cross sections). All of these features are persistent throughout the DL quadrant and thus are present in the quadrant average (Figs. 7d-f). In particular, the descending inflow is a mesoscale feature that contributes to 


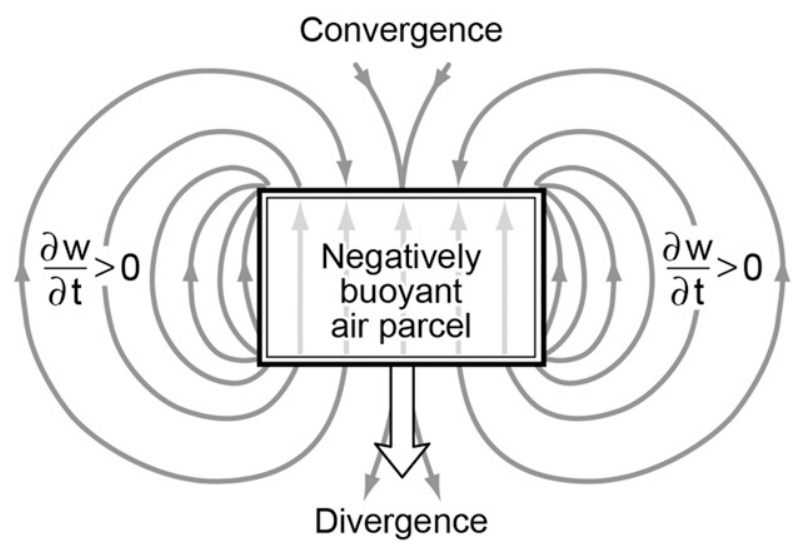

FIG. 9. Schematic illustrating the BPGA field that a negatively buoyant air parcel exerts on its environment as it sinks. Force field lines induce divergence, upward acceleration $(\partial w / \partial t>0)$, and convergence in the indicated areas. Adapted from Didlake and Houze (2009).

the axisymmetric midlevel inflow in Fig. 3b. Also, the tangential wind maximum at updraft $\mathrm{B}$ corresponds to the axisymmetric wind maximum at $125-\mathrm{km}$ radius in Fig. 3b.

Figure 8 shows a cross section (slice 2) $2 \mathrm{~h}$ later and slightly upwind of Fig. 7. Here, stratiform precipitation is prominent outside of $110-\mathrm{km}$ radius. Collocated strong midlevel inflow appears in the outer radii, and then abruptly stops between $140-$ and $160-\mathrm{km}$ radius. This inflow jet occurs between 2- and 4-km altitude, which is just below the bright band as in Fig. 7. At this radius, the inflow jet is collocated with a midlevel tangential wind maximum and convergence maximum. This airflow then descends into the boundary layer, much more abruptly than in slice 1 . But similar to slice 1 , the midlevel convergence also stretches into the boundary layer and connects to an updraft (B) that rises from the boundary layer. Based on this close proximity, it is possible that the descending inflow plays a role in forcing this updraft, as in slice 1. The updraft is not apparent in the DL average, but the mesoscale descending inflow and associated convergence pattern are clearly seen in Figs. 8d,e.

Traveling inward, radial inflow continues in the lowest layers, which is much stronger low-level inflow than that seen in Fig. 7. At $80-100 \mathrm{~km}$, this enhanced radial inflow slows down and connects to another intense updraft (A). This feature, upon closer inspection, is associated with the same updraft at $60-\mathrm{km}$ radius in Fig. 7, also marked updraft A. Both updrafts A in Figs. 7 and 8 are associated with the same convective line also marked $\mathrm{A}$ in Fig. 6b. This convective line has intensified, grown upwind, and propagated radially outward in the 2-h gap between cross sections. The continuous LF radar data indicate that the entire structure (descending inflow, continuing boundary layer inflow, double updrafts, and associated tangential wind maxima) in Fig. 7 is a downwind continuation of Fig. 8 .

The features in Figs. 7 and 8 share many similarities with the mesoscale descending inflow found in Didlake and Houze (2013b). These similarities include being located in the stratiform-dominant DL quadrant, strong midlevel inflow beneath the bright band, descent of the inflow into the boundary layer, and enhanced low-level inflow radially inward. It is likely that a radial buoyancy gradient set up by differential latent heating forces the mesoscale descending inflow here in Earl as it did in Hurricane Rita (Didlake and Houze 2013b).

One notable departure from Didlake and Houze (2013b) is the presence of two updrafts stemming from the boundary layer. We hypothesize that updraft $\mathrm{B}$ is directly connected to the stratiform rainband dynamics. The mesoscale inflow is negatively buoyant because of diabatic cooling, and as a result descends into the boundary layer. This negatively buoyant descent may also be locally enhanced by precipitation drag as described in Didlake and Houze (2013b).

A negatively buoyant air parcel creates a buoyancy pressure gradient acceleration (BPGA) field as shown in Fig. 9. As the air parcel sinks, the surrounding air diverges at the bottom of the parcel, accelerates upward on the sides of the parcel, and converges at the top of the parcel. Supposing that this air parcel is part of the continuous mesoscale descending inflow, then a continuous region of upward acceleration and convergence should occur above and radially inward of the descending inflow. Such convergence fields are seen in Figs. 7b,e and 8 b,e. As the air parcel enters the boundary layer, the equivalent potential temperature $\left(\theta_{e}\right)$ of its surroundings rapidly increases such that the upward acceleration of high $\theta_{e}$ air along the sides of the parcel can more readily become positively buoyant and erupt upward. Also, the horizontal accelerations above the air parcel can produce a nonlinear response where convergence of high $-\theta_{e}$ boundary layer air could also yield a positively buoyant updraft. For either mechanism, the updraft lies along the inner side of the mesoscale descending inflow like that seen in updraft B.

Beneath the air parcel in the descending inflow, the BPGA field induces a negative radial pressure gradient just radially inward of the downdraft, which would locally accelerate the low-level inflow. This is a possible explanation for the enhanced boundary layer inflow in Fig. 8. In idealized simulations, Moon and Nolan (2010) show such an inflow perturbation as a balanced vortex response to low-level cooling (see their Fig. 14b). In a full-physics simulation, Qiu and Tan (2013) also 
Flight 3: $1053 Z$

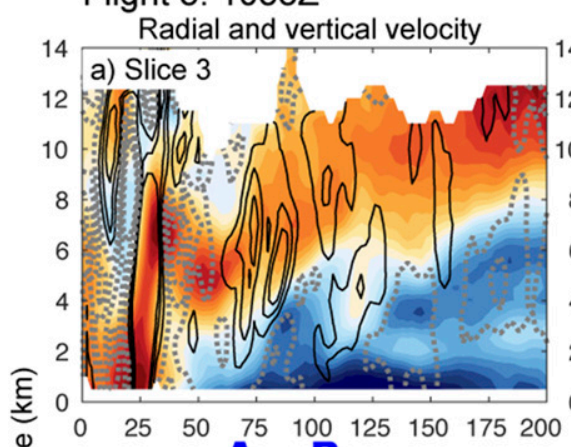

Divergence and vertical velocity Tangential velocity and reflectivity
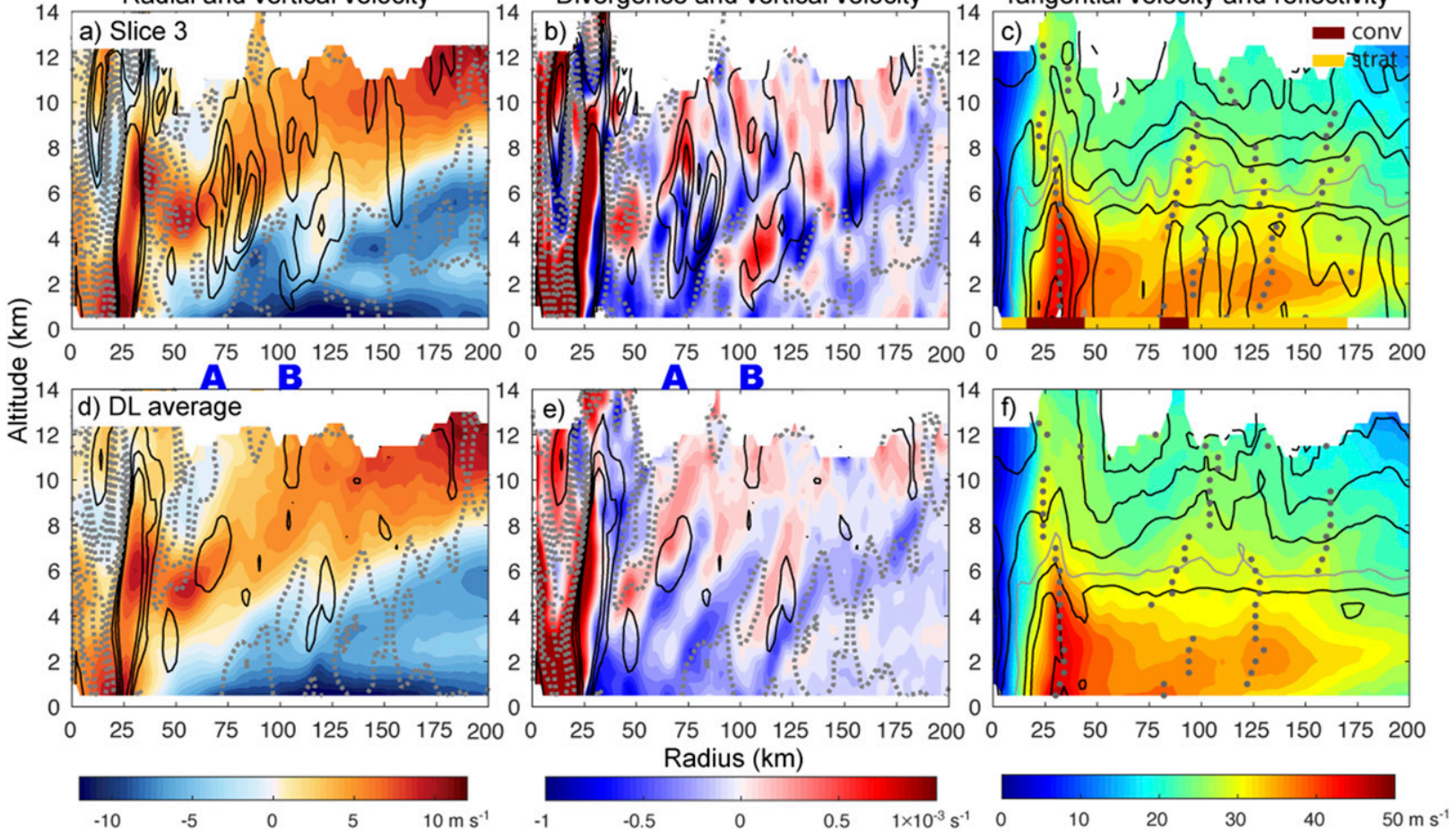

FIG. 10. As in Fig. 7, but for slice 3 and the DL average of flight 3 at 1053 UTC as shown in Fig. 6c. The 30-dBZ reflectivity contour is shown in gray in (c) and (f).

highlight this enhanced boundary layer inflow resulting from pressure perturbations created by a mesoscale descending inflow. In observations, Didlake and Houze (2013b) also show that this boundary layer inflow perturbation occurs radially inward of the mesoscale descending inflow.

The connection between updraft $\mathrm{A}$ and the mesoscale descending inflow is not as readily apparent. Updraft A is certainly a heat source that locally accelerates low-level inflow radially outside of it, as described by Shapiro and Willoughby (1982). Based on the current observations, it is not clear how much of this enhanced inflow is due to updraft $\mathrm{A}$ or the negatively buoyant downdraft from the stratiform dynamics. Qiu and Tan (2013) argued that the enhanced boundary layer inflow was originally driven by the stratiform dynamics. They also posited that this local acceleration caused the gradient of radial velocity to become sharpened and resulted in a narrow zone of boundary layer convergence and persistent updraft. In our case, this could explain updraft A. We do see in the observations that updraft A persistently occurs in the DL quadrant and grows upwind over time from this quadrant. This upwind growth would result from induced convergence upwind in response to downdrafts reaching the boundary layer, in the manner described by Didlake and
Houze (2009) and Sawada and Iwasaki (2010). Based on its association with the DL stratiform precipitation, we hypothesize that updraft A and the mesoscale descending inflow are connected, but such speculation needs to be further examined in future studies.

Figure 10 shows a DL cross section (slice 3 ) and the DL average on the next day from flight 3 indicated in Fig. 6c. In slice 3, stratiform precipitation (Fig. 10c) is collocated with a descending inflow jet that extends from $>200-\mathrm{km}$ radius at $6-\mathrm{km}$ altitude to $125-\mathrm{km}$ radius at $4 \mathrm{~km}$ (Fig. 10a). There are two distinct branches of this descending inflow at different altitudes, where the upper and lower branches likely correspond to different regions of cooling from sublimation and melting, respectively. The jet then descends into the boundary layer near $130-\mathrm{km}$ radius, where just radially inward the boundary layer inflow is locally enhanced. Nearby a local tangential wind maximum exists (Fig. 10c) and its associated enhanced tangential winds extend radially outward. On the radially inward side are regions of convergence that also extend into the boundary layer, and an updraft (B) originates from this point of convergence (Fig. 10b), just as in the previous slices. These similarities suggest that the same mechanisms from flight 2 are operating here. The DL average also exhibits 
a) Flight 2

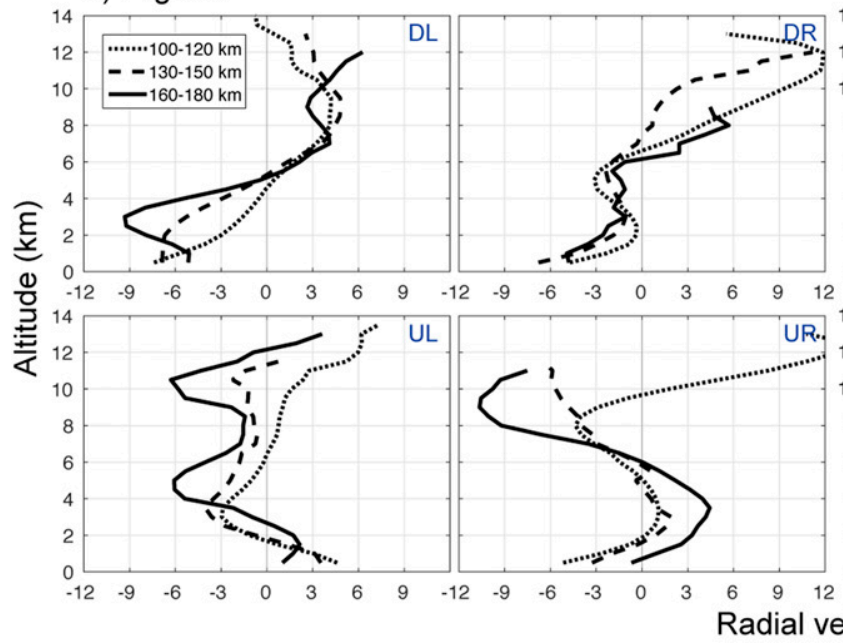

b) Flight 3
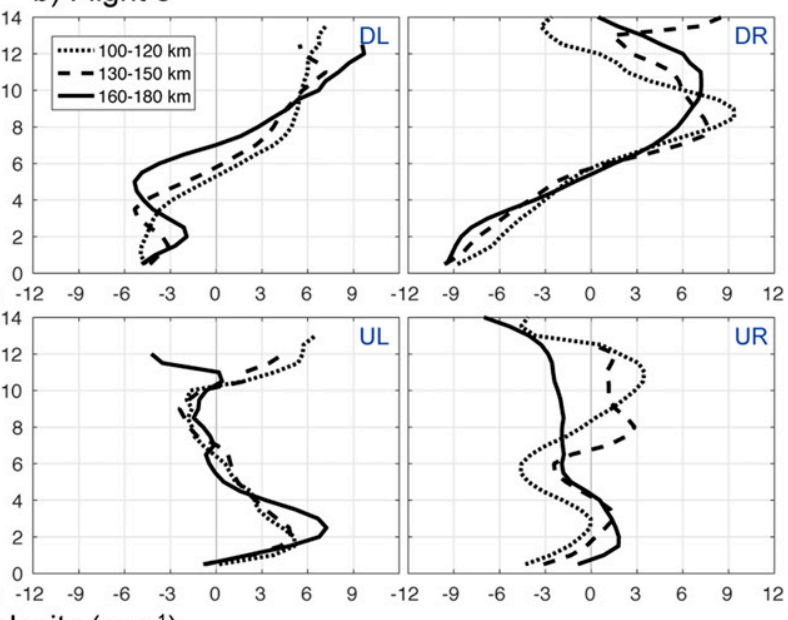

FIG. 11. Vertical profile of radial velocity averaged over selected radial ranges of 100-120 (dotted), 130-150 (dashed), and 160-180 (solid) km in each shear-relative quadrant for (a) flight 2 and (b) flight 3.

most of these same structures, as this overall flow pattern is indeed a mesoscale feature. Updraft B is present in the average and extends to the boundary layer with a magnitude $\left(0.1 \mathrm{~m} \mathrm{~s}^{-1}\right)$ smaller than the contour shown. A second updraft (A) appears near $70-\mathrm{km}$ radius and is associated with a low-level tangential wind maximum near $80-\mathrm{km}$ radius, which is distinct from the maximum near $125-\mathrm{km}$ radius. These two maxima correspond to the axisymmetric tangential wind maxima at the same radii (Fig. 3c). The two updrafts correspond to the convective lines marked in Fig. $6 \mathrm{c}$.

The mesoscale descending inflow is clearly a persistent feature in the DL quadrant. We now search for signs of this pattern in other quadrants. Figure 11 presents the vertical profile of radial wind averaged over specific radial ranges from each quadrant. In flight 2 , the DL quadrant shows an inflow peak descending with decreasing radius, corresponding to the mesoscale descending inflow in Figs. 7 and 8. Looking at the DR quadrant, there is a slight peak in weaker midlevel inflow as well which descends about $0.5 \mathrm{~km}$. The UL quadrant also has a local inflow peak near $5 \mathrm{~km}$, which descends about $2 \mathrm{~km}$. Individual cross sections confirm that both UL and DR quadrants exhibit similarities to the descending inflow jet as in the DL quadrant here (albeit its descent is not as deep). This jet stretches across these three adjacent quadrants, but the DL quadrant is where it is strongest. In flight 3 , the DL quadrant again exhibits a prominent but weaker descending inflow, consistent with Fig. 10. At this point, no other quadrant shows signs of this feature.

\section{b. Tangential wind tendency}

The local tangential wind maxima in all of the DL slices appear dynamically related to the mesoscale descending inflow jet, and most of these local maxima occur at radii of axisymmetric maxima. We now want to learn how important these DL features are relative to other quadrants in shaping the axisymmetric wind field. In particular, by examining the dynamics of these features, we can assess their potential role in building the secondary eyewall that eventually forms in Earl. To perform this analysis, we calculate terms from the tangential momentum equation in cylindrical coordinates $(r, \theta, z)$, which is given by

$$
\frac{\partial v}{\partial t}=-u \eta-\frac{v}{r} \frac{\partial v}{\partial \theta}-w \frac{\partial v}{\partial z}-\frac{1}{\rho r} \frac{\partial p}{\partial \theta}+F_{\theta}
$$

where

$$
\eta=\frac{\partial v}{\partial r}+\frac{v}{r}+f
$$

In these equations, $u, v$, and $w$ are the radial, tangential, and vertical velocities, respectively; $p$ is pressure; $\rho$ is density; and $f$ is the Coriolis parameter. The variable $\eta$ is the combined vertical vorticity resulting from radial shear of the tangential wind, curvature of the tangential wind, and the Coriolis effect. The right-hand-side terms of Eq. (1) represent radial flux of vertical vorticity, azimuthal advection, vertical advection, pressure gradient acceleration, and dissipation. The dissipation and pressure gradient acceleration terms are certainly important to the momentum budget; however, these terms cannot 

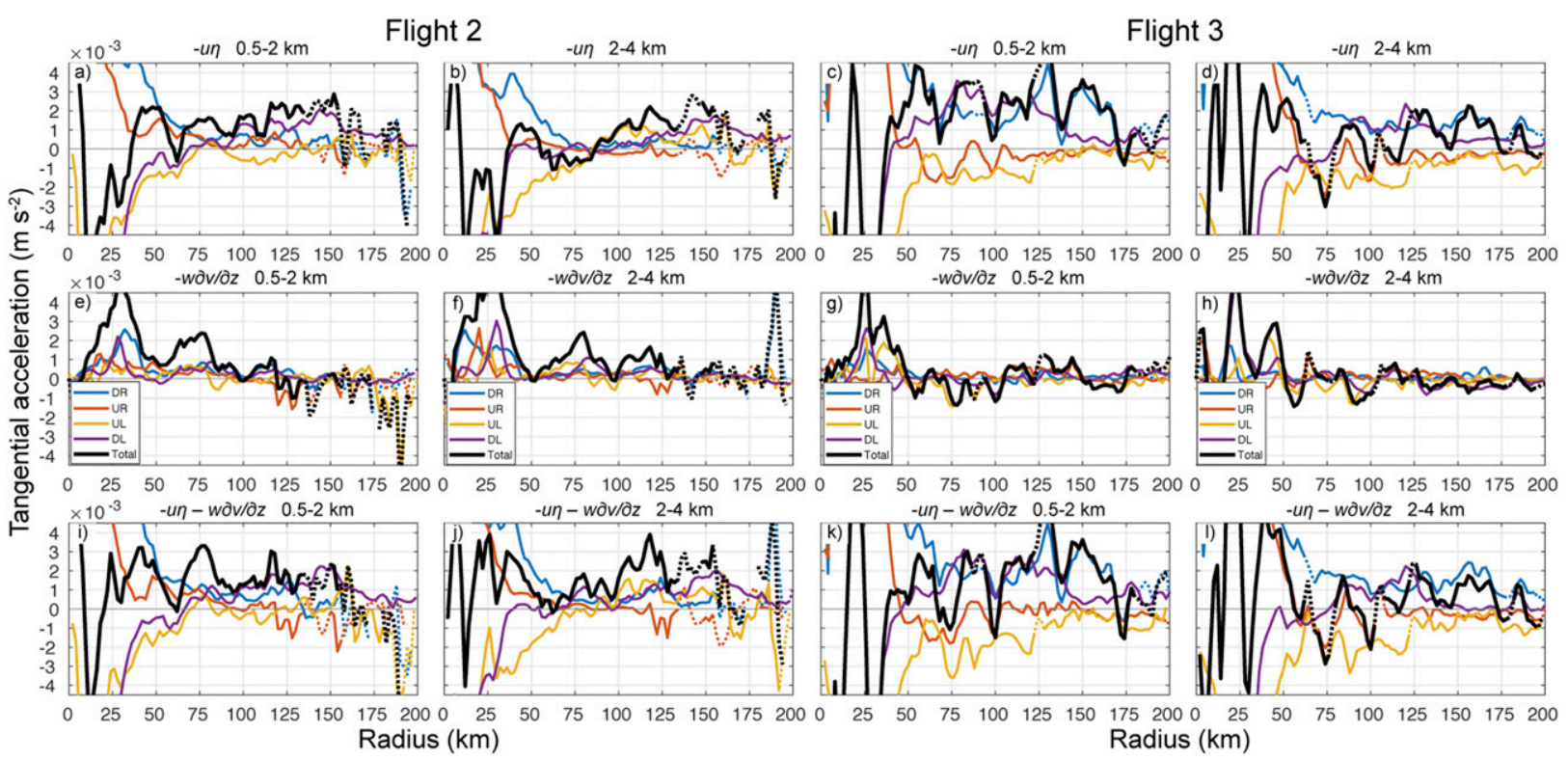

FIG. 12. Radial profiles of terms from the tangential momentum tendency equation averaged over (columns 1 and 3) 0.5-2-km and (columns 2 and 4) 2-4-km altitudes for flight 2 and flight 3. (top to bottom) The radial vorticity flux term, the vertical advection term, and their sum are shown for each shear-relative quadrant and the total of all quadrants: DR (blue), UR (red), UL (yellow), DL (purple), and Total (black). Dashed portions of each line mark locations with a $\geq 45^{\circ}$ gap in the azimuthal data coverage.

be calculated with the current dataset. We refer to the remaining terms as the advective tendency terms. Calculations of the azimuthal advection term should theoretically yield a sum over all quadrants equal to zero. In this dataset, the azimuthal advection term yielded significant variability in profiles for each quadrant and a nonzero sum of all quadrants (not shown). Given the flight pattern of each mission, large gaps can exist between the center-crossing flight legs, particularly at larger radii. These gaps likely caused the erroneous nonzero total sum and created residuals that were large relative to the other terms. To avoid making interpretations based on known sources of error, we ignore azimuthal advection and focus on the radial flux and vertical advection terms in the current analysis.

Figure 12 shows the advective tendency terms and their sum ${ }^{2}$ averaged for all quadrants and totaled over

\footnotetext{
${ }^{2}$ The vertical advection term may be subject to error in the present analysis because of a heavy weighting of the mass continuity equation in the variational wind retrieval technique (Reasor et al. 2009). Since the retrieved vertical wind is effectively an integrated quantity over several surrounding grid points, it will tend to be a smoothed representation of the actual vertical wind field. This suggests general caution in interpreting the sum of budget terms involving vertical wind. Interpretations of the current analysis are carefully made to account for moderate underestimations in the nonzero vertical advection magnitudes.
}

the entire storm for flights 2 and 3. To account for the data coverage issue, data where azimuthal gaps are less than $45^{\circ}$ in each quadrant are shown as a solid line, while locations with larger gaps are shown as a dashed line. In the low levels $(0.5-2-\mathrm{km}$ altitude) for flight 2 , the total azimuthally averaged advective tendency is positive throughout most radii in the rainband region ( $>60-\mathrm{km}$ radius) at both low levels $(0.5-2-\mathrm{km}$ altitude; see Fig. 12i) and middle levels (2-4-km altitude; see Fig. 12j). This signal is consistent with a broadening storm (Fig. 3). Several peaks occur, including at 75-, 120-, and $150-\mathrm{km}$ radius. The peak near $75 \mathrm{~km}$ is primarily due to vertical advection (Figs. 12e,f) and corresponds to updraft A in Figs. 7 and 8. For the other two peaks, the DL and DR have greater advective tendencies than the other quadrants in the low levels (Fig. 12i), and the DL and UL generally have the largest tendencies in the middle levels (Fig. 12j). Radial vorticity flux is the primary contributor to the DL tendency patterns outside of $125 \mathrm{~km}$ (Figs. 12a,b). In particular, these tendencies correspond to the low-level and midlevel impacts of the mesoscale descending inflow seen in Figs. 7, 8, and 11. At the $120-\mathrm{km}$ peak, vertical advection (Figs. 12e,f) does contribute to the total tendency. For the DL, this feature likely corresponds to updraft $\mathrm{B}$.

In flight 3 , much like in flight 2 , the total advective tendency is positive throughout most of the rainband region in the low and middle levels (Figs. 12k,1). The DL 

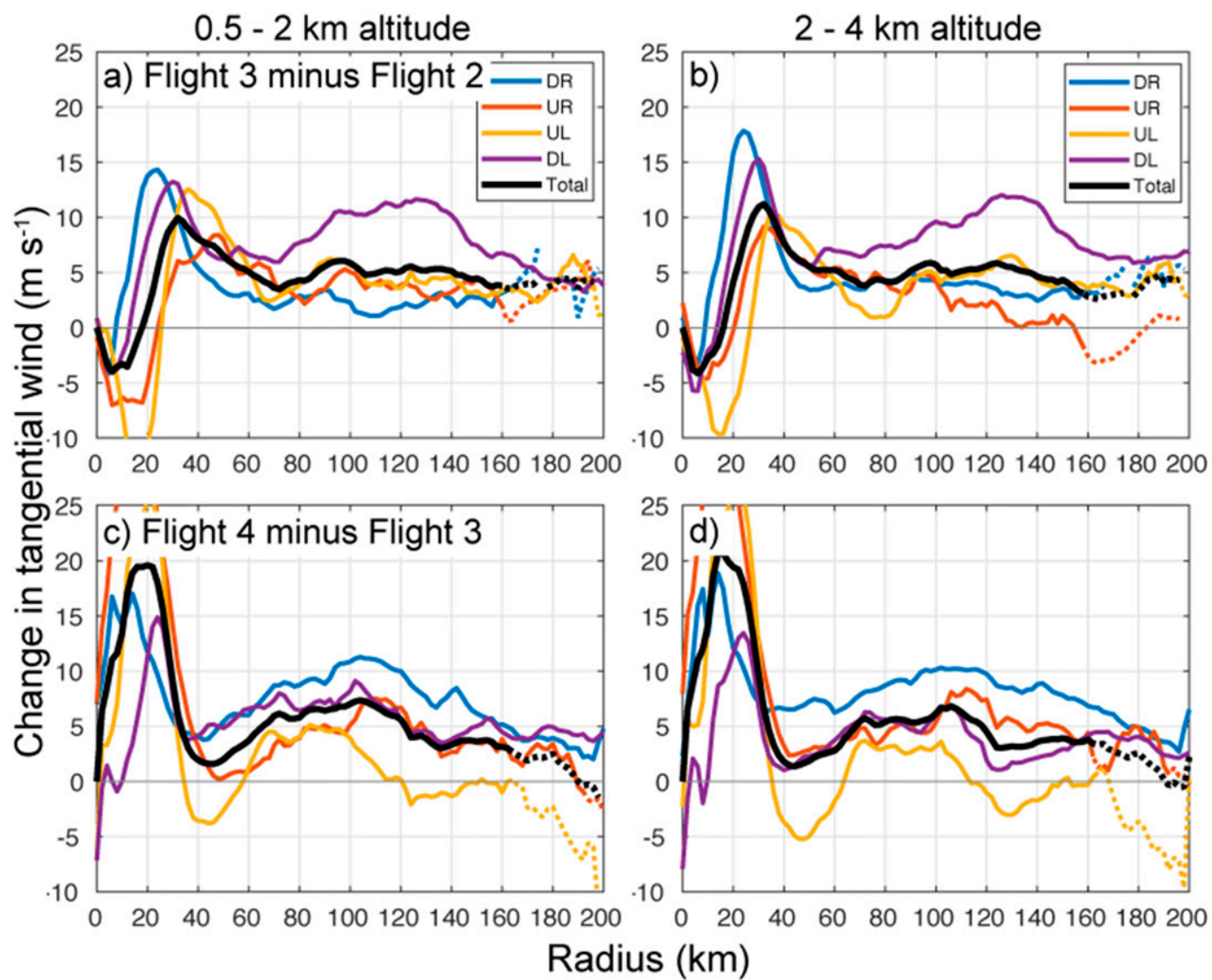

FIG. 13. Difference in the average radial profile of tangential velocity between (a),(b) flights 3 and 2 and (c),(d) between flights 4 and 3 for (a),(c) $0.5-2 \mathrm{~km}$ and (b),(d) $2-4 \mathrm{~km}$ altitude. Differences are taken of averaged shear-relative quadrants and of the total azimuthal average within the indicated altitudinal layers.

and DR quadrants have the larger advective tendencies throughout, and radial vorticity flux is the primary contributor to the total. The DR increase in radial vorticity flux from flight 2 to flight 3 is consistent with the clear increase in low-level inflow seen in Fig. 11. This change is likely related to the increased rainband convection in Fig. 1c that lies to the right of the shear vector. A local maximum occurs in the DL at $120-\mathrm{km}$ radius for both low and middle levels (Figs. 12c,d). These features of enhanced radial vorticity flux correspond to the inner edge of the mesoscale descending inflow in Fig. 10d.

We now compare the instantaneous tendency calculations in Fig. 12 to the actual evolution of the tangential wind field in between aircraft missions. Figure 13 presents the change in low-level tangential winds between flights 2 and 3 and in between flights 3 and 4 for each quadrant and the total storm. The peaks in the azimuthally averaged advective tendency in Figs. 12i-1 do not match the peaks of the tangential wind evolution in Fig. 13, which indicates that the rainband dynamics did not remain constant during the $\sim 12 \mathrm{~h}$ between flights.
Still, there was some consistency with the quadrantrelative impact on the wind evolution. In Figs. 13a,b (flight 3 minus flight 2), the largest change for both the low and middle levels occurred in the DL quadrant throughout all of the available rainband regions. This large increase compared to other quadrants is consistent with the larger tendency calculated for the DL quadrant at the beginning of this time span (Figs. 12i,j). The peak in the DL change occurs at 130 -km radius, which is radially inward of the outermost $(\sim 150-\mathrm{km}$ radius $)$ peak in the DL tendency. In Figs. 13c,d (flight 4 minus flight 3), the DR quadrant has the largest tangential wind increases throughout the rainband region, while the next largest increase occurs in either the DL or UR, depending on the radius. These changes do not match the tendencies shown Figs. 12k and 12l, where the DL and DR are consistently higher. This discrepancy suggests that tangential wind tendencies are changing between flights 3 and 4, most noticeably in the UR quadrant. Returning to the DL quadrant, its peak in tangential wind change occurs near $105-\mathrm{km}$ radius, which is again radially 

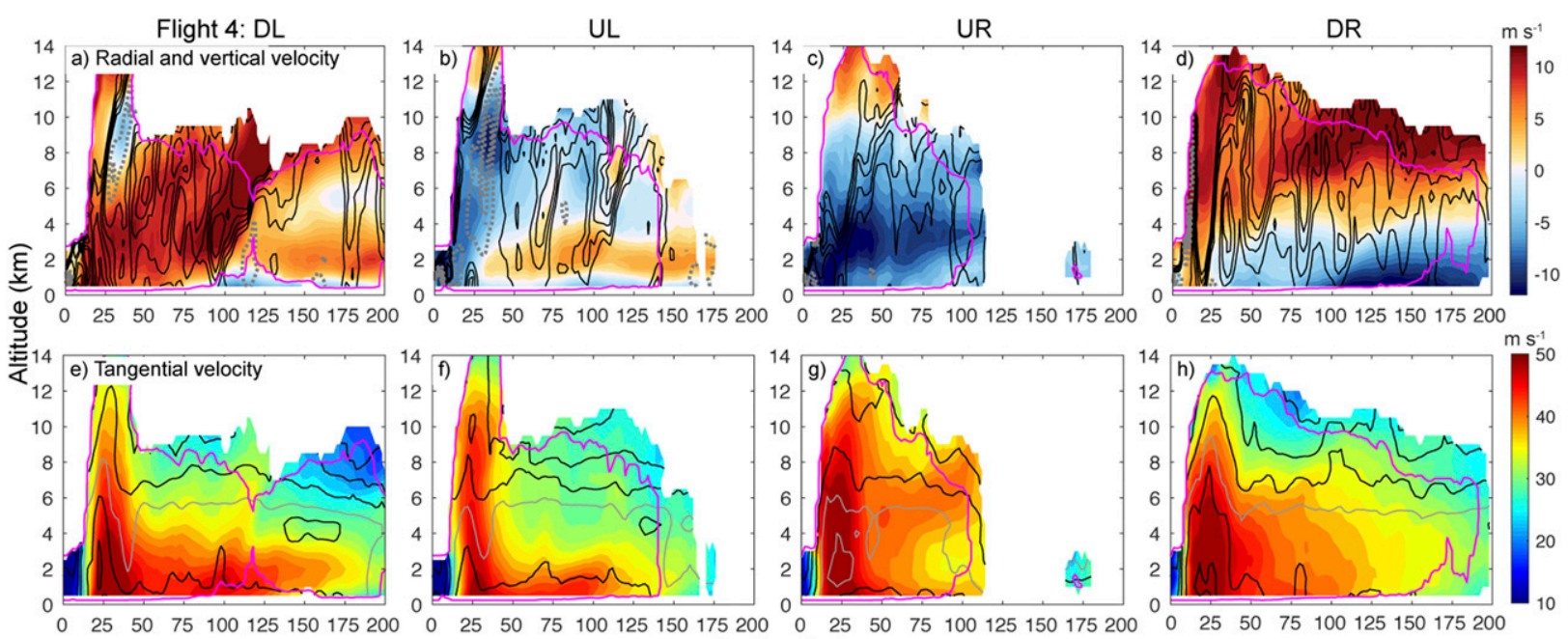

Radius (km)

FIG. 14. As in Fig. 7, but for averaged cross sections in each shear-relative quadrant of flight 4. The 30-dB $Z$ reflectivity contour is shown in gray in (e)-(h).

inward of the outermost $(\sim 120-\mathrm{km}$ radius $)$ peak in the DL tendency.

Despite the offset of time spans and differences in indicators of tangential wind acceleration, this joint analysis produces a consistent story for the DL quadrant. The tangential wind tendencies in the DL quadrant, which are largely shaped by the radial vorticity flux term, are shown to be significant to the overall total and have peaks that correspond to the dynamics of the mesoscale descending inflow. Radially inward (15-20 km) of these peaks, the DL tangential winds substantially increased afterward. If a tendency maximum contracted inward over time, then subsequent wind increases would occur radially inward of the initial tendency maximum. Based on these results, we hypothesize that the DL peaks in tangential wind change (Fig. 13) are directly resulting from a contracting stratiform rainband with mesoscale descending inflow (and its associated updraft B) that accelerates the tangential winds at its innermost extent.

\section{c. Flight 4}

Leading into flight 4 , the axisymmetric tangential winds in the rainband region had its largest increase at $110-\mathrm{km}$ radius (Figs. 13c,d). This maximum increase was a result of contributions from the DR, DL, and UR quadrants. We now examine the asymmetric wind structures that resulted from these quadrant changes. Figure 14 displays quadrant averages of the wind field during flight 4 .

In the DL quadrant, a clear tangential wind maximum of $48 \mathrm{~m} \mathrm{~s}^{-1}$ occurs at $100-\mathrm{km}$ radius and $1.5-\mathrm{km}$ altitude. This location aligns with the outer wind maximum in Fig. 3d, showing that this DL maximum projects strongly onto the axisymmetric average. Associated with this wind maximum is a prominent channel of rising outflow, with radial velocities greater than $10 \mathrm{~ms}^{-1}$ and updrafts greater than $4 \mathrm{~m} \mathrm{~s}^{-1}$. This airflow channel appears similar to the secondary eyewall overturning circulation seen in Hurricane Rita (Houze et al. 2007; Didlake and Houze 2011). As in Rita, the outflow channel here in Earl begins with a near-horizontal layer of radial outflow in the lower levels $(2-3.5 \mathrm{~km})$. This channel then makes an upward turn that continues into the upper levels. The tangential wind and secondary circulation structure here appear to be that of the developing secondary eyewall of Earl.

The location of the DL wind maximum closely matches the DL peak in tangential wind increase (Fig. 13b), which was hypothesized to be a direct result of the mesoscale descending inflow. This suggests that the DL wind maximum leading to a secondary eyewall was created by the DL mesoscale descending inflow in the previous $\sim 24 \mathrm{~h}$. Compared to previous flights, the mesoscale descending inflow is noticeably missing in Fig. 14a. Some inflow and weakened outflow exist between 4 and $6 \mathrm{~km}$ outside of the developing secondary eyewall. This pattern appears to slant downward with decreasing radius, and could be remnants of the mesoscale descending inflow. In the processes described here, the mesoscale descending inflow would have to develop the DL wind maximum and induce a sustained updraft (via interaction of the negatively buoyant air and the boundary layer) prior to its dissipation shown by Fig. 14a.

Downwind in the UL quadrant, the secondary wind maximum is slightly weaker at $44 \mathrm{~m} \mathrm{~s}^{-1}$ and radially inward ( $\sim 90-\mathrm{km}$ radius). Portions of the DL secondary 
eyewall channel extend into this quadrant. Enhanced radial outflow between 80 and $100 \mathrm{~km}$ is connected to an updraft that reaches the upper levels. In the rainband region of the UR quadrant, enhanced tangential winds occur in the middle levels $(4-8 \mathrm{~km})$ and below $1 \mathrm{~km}$. The low-level winds are weaker than in the left-of-shear quadrants, and there is no clear secondary wind maximum between 80 - and $90-\mathrm{km}$ radius.

The DR quadrant has stronger tangential winds throughout the rainband region than in the UR quadrant, which is consistent with the tangential wind tendencies in Fig. 12. Yet, the low-level wind magnitudes do not match those of the UL and DL quadrants, and there is no apparent wind maximum between 80 - and $100-\mathrm{km}$ radius. The secondary circulation does indicate strong low-level radial convergence leading into two updrafts at $80-$ and $100-\mathrm{km}$ radius, which extend into the upper levels and continues outward as strong radial outflow. This overturning circulation pattern occurs at the radius expected of the developing secondary eyewall. However, this circulation pattern is not outwardly slanted and is not associated with a low-level tangential wind maximum that is observed in an axisymmetric secondary eyewall. The DL quadrant has all of the expected secondary eyewall features, which suggests that this quadrant plays some initiating role in the secondary eyewall developing in Earl. The dynamical consequence of the mesoscale descending inflow in DL stratiform rainband precipitation is a plausible mechanism for initiating the secondary eyewall.

The reflectivity pattern seen in Fig. $6 \mathrm{~d}$ shows that the strongest reflectivity in the developing secondary eyewall region occurs in the DL quadrant, being associated with the robust overturning circulation here. The maximum reflectivity of the inner eyewall occurs in the DR quadrant, consistent with the strongest inner eyewall updraft (Fig. 14d). This azimuthal offset of maximum updrafts and reflectivity quadrants between concentric eyewalls is a pattern consistent with previous studies (Hence and Houze 2012a; Didlake et al. 2017).

\section{WSR-88D observations of Earl}

The axisymmetric average of flight 4 (Figs. 3d and 4d) revealed two tangential wind maxima and associated overturning circulations between $60-80$ and $80-100 \mathrm{~km}$. We have shown that the outermost feature was potentially formed by dynamics of the mesoscale descending inflow (and the associated updraft B in Figs. 7, 8, and 10) occurring in the DL stratiform rainband. We have also highlighted this outermost feature as the initial signature of a developing secondary eyewall, thereby connecting updraft B directly to the secondary eyewall. On the other

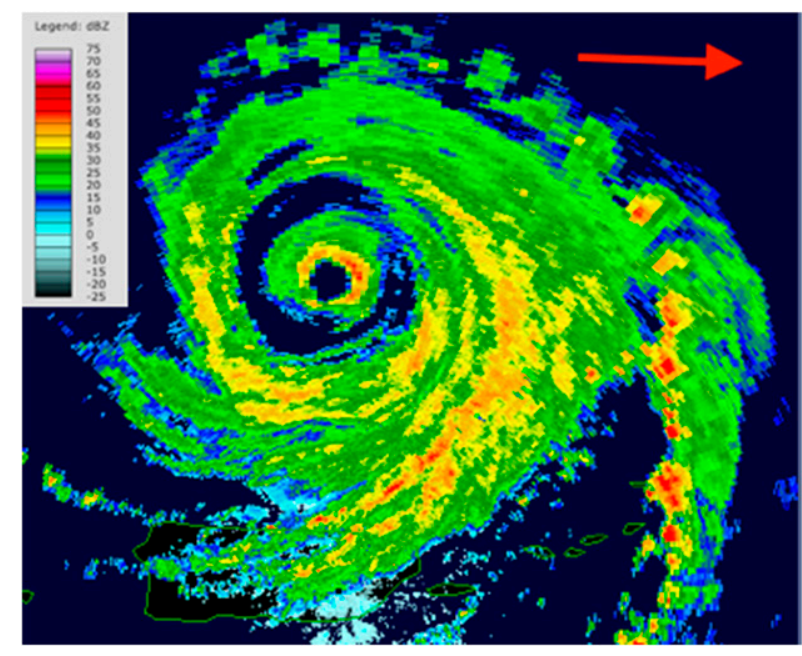

FIG. 15. Reflectivity (dBZ) from the lowest elevation scan of the TJUA WSR-88D taken at 0437 UTC 31 Aug 2010. The environmental wind shear vector is shown in red. Image comes from the NOAA Weather and Climate Toolkit.

hand, the second, innermost updraft in flights 2-3 (updraft A) was not clearly associated with the dynamics that led to secondary eyewall development. For flight 4 , it is not yet apparent if the observed innermost feature $(60-80 \mathrm{~km})$ here is associated with the developing secondary eyewall. To further examine the source features for the eventual secondary eyewall, we explore WSR-88D observations that provide time continuous data during these crucial stages of secondary eyewall formation.

The center of Hurricane Earl tracked in range of the San Juan radar at 1700 UTC 30 August. Figure 15 shows the low-level reflectivity at 2226 UTC 30 August. A clear secondary eyewall can be seen in the reflectivity pattern. We applied the GBVTD (Lee et al. 1999) analysis to the WSR-88D data to retrieve the axisymmetric tangential winds and track the development of Earl's secondary eyewall. Unfortunately, the storm center remained a significant distance from the radar $(\sim 100 \mathrm{~km}$ on average) during its time in range. With Earth's curvature and the lowest radar elevation angle of $0.4^{\circ}$, the lowest level of analyzed winds is 4-km altitude. At this level, though, both outer tangential maxima (Fig. 3d) should still be captured by the analysis.

Figure 16 shows a Hovmöller diagram of the $4-\mathrm{km}$ axisymmetric tangential winds in Earl. Wind data are only available where precipitation echoes sufficiently fill the analyzed radial ring. The inner eyewall can be seen clearly and consistently between $15-$ and $35-\mathrm{km}$ radius. Its tangential winds strengthen over time, reaching $55 \mathrm{~m} \mathrm{~s}^{-1}$. Compared to the $\mathrm{P} 3 \mathrm{TDR}$ analysis and C-130 flight level observations, the GBVTDderived wind maximum were $\sim 5 \mathrm{~m} \mathrm{~s}^{-1}$ higher. This 
overestimation is often seen in GBVTD analyses and is likely due to errors in the initial assumptions. Still, this $10 \%$ error falls within the acceptable theoretical range (Lee et al. 1999).

Outside of the inner eyewall, the rainband signal is more variable prior to 2100 UTC 30 August. This is a result of rainbands being more asymmetric and also less likely to adequately fall within range of the radar. By 2100 UTC, the rainband signal extends beyond $80-\mathrm{km}$ radius. At 2200 UTC, a secondary wind maximum (marked by an $\times$ ) reaching $44 \mathrm{~m} \mathrm{~s}^{-1}$ develops between 50 - and $65-\mathrm{km}$ radius. The timing and location of this maximum is consistent with the $60-80-\mathrm{km}$ maximum and corresponding overturning circulation in flight 4 (Figs. 3d and 4d).

By 0000 UTC 31 August, the winds at this radius have decreased sharply and the axisymmetric signal of precipitation declines. A moat region characteristic of concentric eyewalls becomes apparent between 35- and $60-\mathrm{km}$ radius indicating a lack of scatterers, while at larger radii, a secondary ring of precipitation forms. The wind magnitudes between $80-$ and $100-\mathrm{km}$ radius steadily increase in the next $4 \mathrm{~h}$, often appearing slightly greater than the winds just radially inward. The dotted line in Fig. 16 follows the approximate locations of this local tangential wind maximum, beginning at 0100 UTC near $90-\mathrm{km}$ radius. This wind maximum is about $10 \mathrm{~km}$ radially inward and $1.5 \mathrm{~h}$ after the outer wind maximum in Fig. 3d. With such close proximity in time and space, it is likely that these two signals are from the same feature, where discrepancies could result from the different observational and analysis methods. Between 0100 and 0300 UTC, this wind maximum strengthens slightly but does not steadily contract over time as predicted by theoretical models. Fluctuations in the radius of secondary wind maximum are not uncommon (Sun et al. 2013). By 0500 UTC, the secondary wind maximum is located at $78-\mathrm{km}$ radius and is positioned to become the outer wind maximum in Fig. 5e approximately $11 \mathrm{~h}$ later.

The GBVTD analysis shows that the axisymmetric tangential wind maximum at $90-\mathrm{km}$ radius on 0100 UTC contracts over time and becomes the wind maximum of Earl's secondary eyewall. This feature is not linked to the midlevel wind maximum near $60-\mathrm{km}$ radius. The $90-\mathrm{km}$ radius wind maximum appears to be the same wind maximum near $80-100 \mathrm{~km}$ seen in flight 4 , which is associated with an overturning circulation expected of a secondary eyewall. This feature is most robust in the DL quadrant, which suggests that the dynamics of the mesoscale descending inflow and updraft B in the DL are critical for its development in earlier stages.

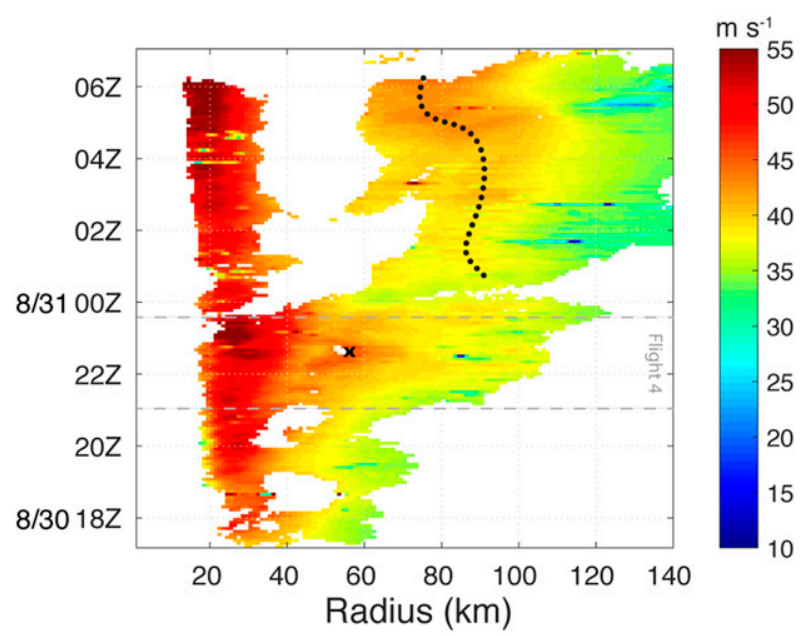

FIG. 16. Azimuthal average of the 4-km-level tangential velocity radial profile over time derived from the GBVTD algorithm. The dotted line marks the approximate location of a local tangential wind maximum associated with Earl's developing secondary eyewall. The $\times$ marks another local maximum associated with innercore rainbands. The time span of flight 4 is marked by the two thin gray lines.

\section{Conclusions}

The NOAA P3 Tail Doppler radar documented the inner core of Hurricane Earl during the early stages of secondary eyewall formation. We analyzed the kinematic and precipitation structures of inner-core asymmetric rainbands to understand their dynamical role in creating the axisymmetric secondary eyewall. During the rapid intensification phase of Earl's maximum eyewall winds, rainband activity continually increased and organized into a broad stationary band complex typical of a sheared TC. Concurrently, the tangential winds in the rainband region intensified, expanding the wind field of the storm. An axisymmetric secondary wind maximum and overturning circulation developed near 90-km radius, which soon after led to a distinct secondary eyewall.

Our analysis of the storm relative to the environmental wind shear shows that the downshear-left (DL) quadrant exhibited notable features during the transition from inner-core rainbands to a secondary eyewall. These features are shown in the conceptual models in Figs. 17 and 18. Figure 17a shows a plan view of inner-core TC features during an early stage of secondary eyewall formation (SEF). Figure 17b illustrates the change of these inner-core features hours later when a developing secondary eyewall can be seen. Figure 18 displays a cross section through the rainband complex in the DL quadrant. The primary findings are as follows: 
a)

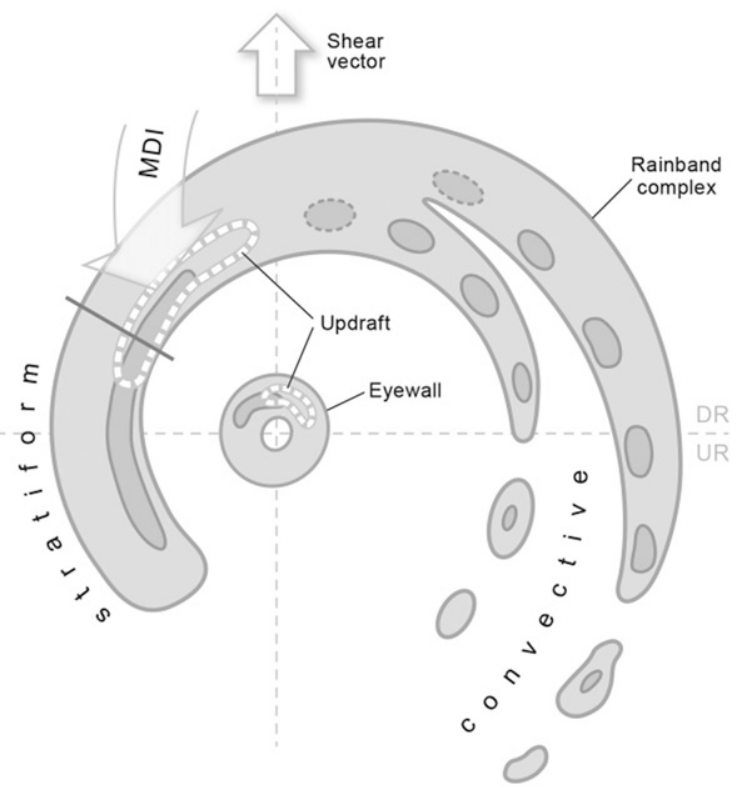

b)

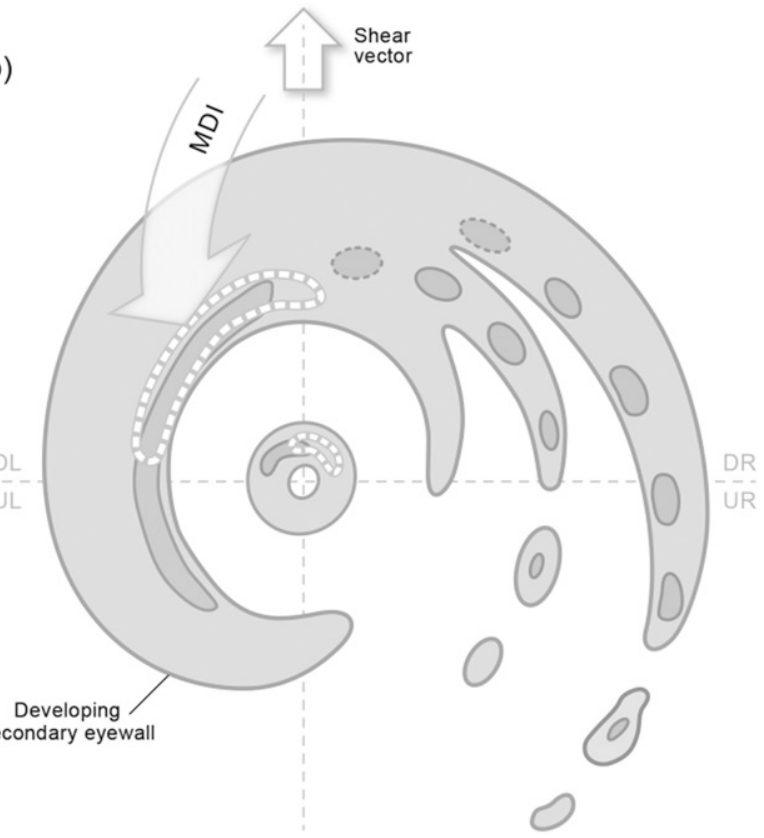

FIG. 17. Plan view schematic of rainband and eyewall structures at two stages of secondary eyewall development: (a) the early stage with a singular eyewall and a spiral rainband complex present, and (b) the later stage with a developing circular ring of secondary eyewall convection. The environmental wind shear vector points upward and defines the four storm quadrants. Reflectivity contours ( 20 and $35 \mathrm{dBZ}$ ) show embedded convective cells in the rainband complex that collapse (gray dashes) and form stratiform precipitation traveling around the storm. MDI and an enhanced updraft (white dashes) both occur in the DL stratiform rainband, and the collocated gray line marks the cross section in Fig. 18. Adapted from Didlake and Houze (2013b).
- A stream of mesoscale descending inflow (MDI) persistently occurred in stratiform rainband precipitation in the DL quadrant, as seen in Fig. 17a. The MDI originated at larger radii ( $>150-\mathrm{km}$ radius), flowed inward for more than $50 \mathrm{~km}$, and then descended into the boundary layer. The MDI was likely a response to radial buoyancy gradients, consistent with that observed in Hurricane Rita (Didlake and Houze 2013b). The cross section in Fig. 18 illustrates the MDI flow pattern.

- Latent cooling from melting and evaporation caused the MDI air to become negatively buoyant $(B<0)$ and descend. The buoyancy pressure gradient acceleration (BPGA) field generated by this negatively buoyant air produced a region of convergence and upward acceleration $(\partial w / \partial t>0)$ that stretched along the inner side of the MDI and into the boundary layer.

- A recurring updraft (updraft B from section 4) occurred at the terminus of the MDI. This updraft was likely triggered by the region of convergence and upward acceleration entering the boundary layer inflow of high $-\theta_{e}$ air.

- The MDI and adjacent updraft produced tangential wind acceleration (indicated by plus signs in Fig. 18) in the radial range where a secondary wind maximum (indicated by developing $V_{T}$ in Fig. 18) and secondary eyewall soon developed.

- Radially inward of the MDI and adjacent updraft lied enhanced boundary layer inflow and a persistent second updraft (updraft A from section 4). These features also accelerated the tangential wind field but they were not evidently connected to the developing secondary eyewall.

- During early signs of the axisymmetric secondary eyewall, the DL quadrant exhibited the strongest overturning circulation and low-level tangential wind maximum. These same features of the primary eyewall occurred upwind (DR quadrant), having an azimuthal offset consistent with that observed in Hurricane Gonzalo (Didlake et al. 2017). Figure 17b illustrates this offset of updrafts and reflectivity between the two eyewalls. The MDI had notably subsided at this point.

The results of this paper confirm the repeatable occurrence of two prominent inner-core features: the MDI in a mature spiral rainband complex and the enhanced DL circulation in a developing secondary eyewall. These results also suggest a plausible dynamical pathway connecting the two features. The MDI, located in the DL quadrant due to the interaction of the spiral band complex with the environmental shear, persistently perturbs the boundary layer flow, which locally forces enhanced convection and tangential winds. 


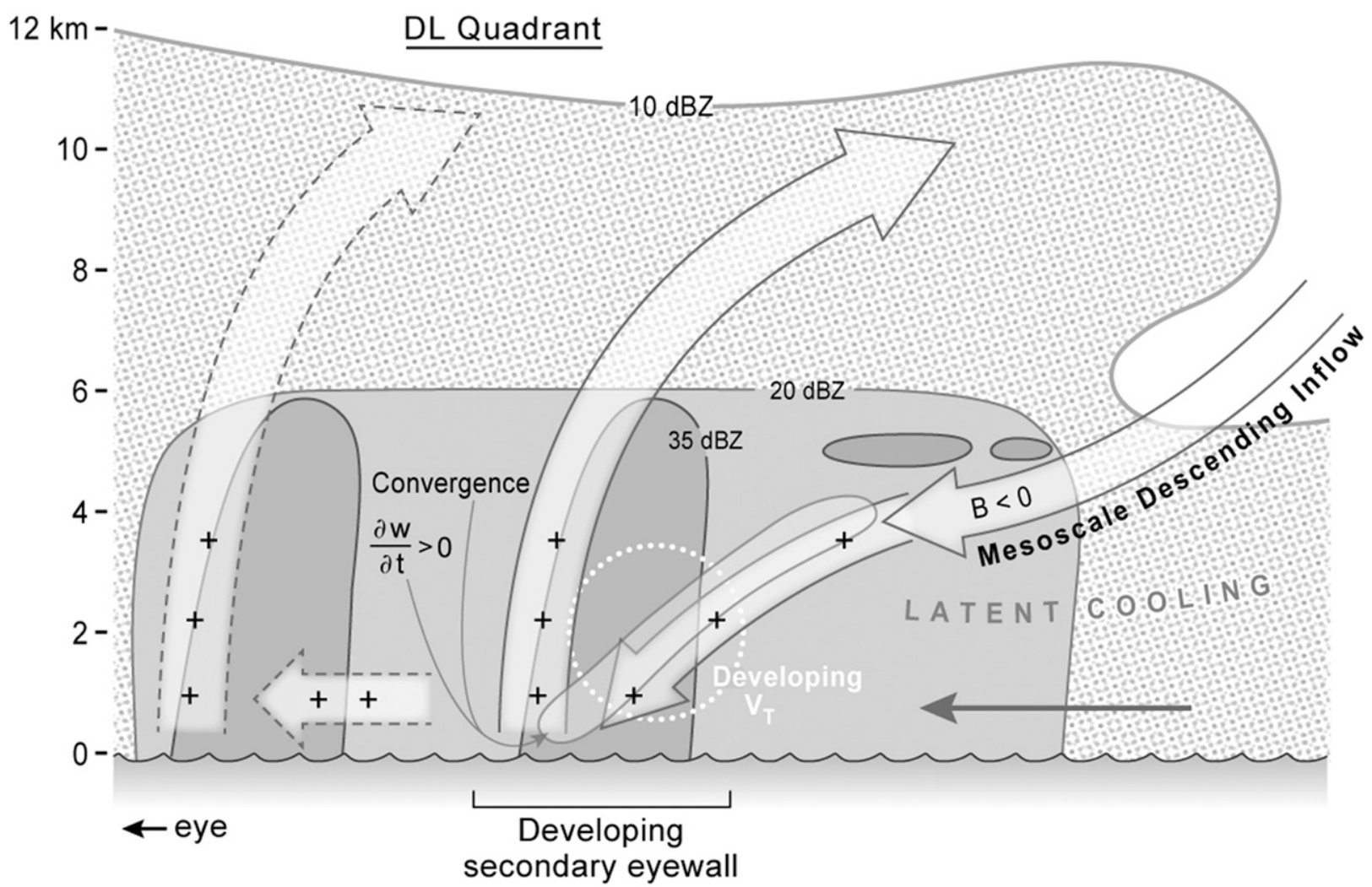

FIG. 18. Cross section schematic of the kinematics within the DL quadrant stratiform rainband at an early stage of secondary eyewall development (see the gray line in Fig. 17). Reflectivity contours are drawn. The solid line arrow shows vortex-scale boundary layer inflow, and the broad solid arrows show motions associated with the stratiform rainband. MDI occurs in a region of latent cooling and is negatively buoyant $(B>0)$. At its end, a convective updraft occurs. Convergence and upward acceleration $(\partial w / \partial t>0)$ lie along the inner side of the MDI. The broad dashed arrows represent additional recurring features: enhanced low-level inflow and a second convective updraft. The plus signs indicate regions of increasing tangential velocity by the secondary circulation. The white-dashed-circled region indicates the location of a developing tangential jet $\left(V_{T}\right)$, which is located in the radial range of the developing secondary eyewall. Adapted from Didlake and Houze (2013b).

Such enhancement in the DL quadrant forges the way for secondary eyewall formation through interaction with the axisymmetric dynamics.

More work is certainly needed to examine the hypothesized relationship among TC inner-core features in the DL quadrant. Such work would be an examination of additional observations of TC inner cores at both high spatial and temporal resolution. The relationship between the MDI and enhanced secondary eyewall convection needs to be further validated. Other rainband processes may also contribute to SEF dynamics. The relative importance of the MDI and processes not discussed in this study needs to be investigated. High-resolution model simulations are critical for examining the dynamics governing the evolution of these observed features.

Acknowledgments. We thank John Gamache for his efforts in developing and maintaining the Tail Doppler radar analysis dataset at HRD. We thank Jonathan
Zawislak for providing the analyzed satellite microwave dataset, and Chau Lam Yu for performing further analysis on this dataset. We thank Beth Tully for her work on the conceptual models. Last, we thank the three anonymous reviewers whose comments led to significant improvements in the manuscript. This research was supported by the NASA New Investigator Program (NIP) under Grant NNX16AI21G.

\section{REFERENCES}

Abarca, S. F., and K. L. Corbosiero, 2011: Secondary eyewall formation in WRF simulations of Hurricanes Rita and Katrina (2005). Geophys. Res. Lett., 38, L07802, https://doi.org/10.1029/ 2011 GL047015.

, and M. T. Montgomery, 2013: Essential dynamics of secondary eyewall formation. J. Atmos. Sci., 70, 3216-3230, https://doi.org/10.1175/JAS-D-12-0318.1.

, and - 2014: Departures from axisymmetric balance dynamics during secondary eyewall formation. J. Atmos. Sci., 71, 3723-3738, https://doi.org/10.1175/JAS-D-14-0018.1. 
Bell, M. M., M. T. Montgomery, and W.-C. Lee, 2012: An axisymmetric view of concentric eyewall evolution in Hurricane Rita (2005). J. Atmos. Sci., 69, 2414-2432, https://doi.org/ 10.1175/JAS-D-11-0167.1.

Black, M. L., and H. E. Willoughby, 1992: The concentric eyewall cycle of Hurricane Gilbert. Mon. Wea. Rev., 120, 947-957, https://doi.org/10.1175/1520-0493(1992) $120<0947$ :TCECOH $>2.0 . \mathrm{CO} ; 2$.

Cangialosi, J. P., 2010: Tropical Cyclone Report: Hurricane Earl. National Hurricane Center Tropical Cyclone Rep. AL072010, 29 pp., https://www.nhc.noaa.gov/data/tcr/AL072010_Earl.pdf .

Corbosiero, K. L., J. Molinari, A. R. Aiyyer, and M. L. Black, 2006: The structure and evolution of Hurricane Elena (1985). Part II: Convective asymmetries and evidence for vortex Rossby waves. Mon. Wea. Rev., 134, 3073-3091, https://doi.org/10.1175/MWR3250.1.

DeMaria, M., M. Mainelli, L. K. Shay, J. A. Knaff, and J. Kaplan, 2005: Further improvements to the Statistical Hurricane Intensity Prediction Scheme (SHIPS). Wea. Forecasting, 20, 531-543, https://doi.org/10.1175/WAF862.1.

Didlake, A. C., Jr., and R. A. Houze Jr., 2009: Convective-scale downdrafts in the principal rainband of Hurricane Katrina (2005). Mon. Wea. Rev., 137, 3269-3293, https://doi.org/10.1175/ 2009MWR2827.1.

$\longrightarrow$, and - 2011: Kinematics of the secondary eyewall observed in Hurricane Rita (2005). J. Atmos. Sci., 68, 1620-1636, https://doi.org/10.1175/2011JAS3715.1.

— and - 2013a: Convective-scale variations in the inner-core rainbands of tropical cyclones. J. Atmos. Sci., 70, 504-523, https://doi.org/10.1175/JAS-D-12-0134.1.

$\longrightarrow$, and 2013b: Dynamics of the stratiform sector of a tropical cyclone rainband. J. Atmos. Sci., 70, 1891-1911, https://doi.org/10.1175/JAS-D-12-0245.1.

—, G. M. Heymsfield, P. D. Reasor, and S. R. Guimond, 2017: Concentric eyewall asymmetries in Hurricane Gonzalo (2014) observed by airborne radar. Mon. Wea. Rev., 145, 729-749, https://doi.org/10.1175/MWR-D-16-0175.1.

Dodge, P., R. W. Burpee, and F. D. Marks Jr., 1999: The kinematic structure of a hurricane with sea level pressure less than $900 \mathrm{mb}$. Mon. Wea. Rev., 127, 987-1004, https://doi.org/ 10.1175/1520-0493(1999)127<0987:TKSOAH>2.0.CO;2.

Fang, J., and F. Zhang, 2012: Effect of beta-shear on simulated tropical cyclones. Mon. Wea. Rev., 140, 3327-3346, https://doi.org/10.1175/ MWR-D-10-05021.1.

Gamache, J. F., 1997: Evaluation of a fully three-dimensional variational Doppler analysis technique. Preprints, 28th Conf. on Radar Meteorology, Austin, TX, Amer. Meteor. Soc., 422-423.

Gao, J., M. Xue, A. Shapiro, and K. K. Droegemeier, 1999: A variational method for the analysis of three-dimensional wind fields from two Doppler radars. Mon. Wea. Rev., 127, 2128-2142, https://doi.org/ 10.1175/1520-0493(1999)127<2128:AVMFTA>2.0.CO;2.

Hence, D. A., and R. A. Houze Jr., 2012a: Vertical structure of tropical cyclones with concentric eyewalls as seen by the TRMM Precipitation Radar. J. Atmos. Sci., 69, 1021-1036, https://doi.org/10.1175/JAS-D-11-0119.1.

$\longrightarrow$, and $-2012 \mathrm{~b}$ : Vertical structure of tropical cyclone rainbands as seen by the TRMM Precipitation Radar. J. Atmos. Sci., 69, 2644-2661, https://doi.org/10.1175/JAS-D-11-0323.1.

Hill, K. A., and G. M. Lackmann, 2009: Influence of environmental humidity on tropical cyclone size. Mon. Wea. Rev., 137, 32943315, https://doi.org/10.1175/2009MWR2679.1.

Hogsett, W., and D.-L. Zhang, 2009: Numerical simulation of Hurricane Bonnie (1998). Part III: Energetics. J. Atmos. Sci., 66, 2678-2696, https://doi.org/10.1175/2009JAS3087.1.
Houze, R. A., Jr., 2004: Mesoscale convective systems. Rev. Geophys., 42, RG4003, https://doi.org/10.1029/2004RG000150.

- S. S. Chen, B. F. Smull, W.-C. Lee, and M. M. Bell, 2007: Hurricane intensity and eyewall replacement. Science, $\mathbf{3 1 5}$, 1235-1239, https://doi.org/10.1126/science.1135650.

Huang, Y.-H., M. T. Montgomery, and C.-C. Wu, 2012: Concentric eyewall formation in Typhoon Sinlaku (2008). Part II: Axisymmetric dynamical processes. J. Atmos. Sci., 69, 662-674, https://doi.org/10.1175/JAS-D-11-0114.1.

Jorgensen, D. P., and J. D. DuGranrut, 1991: A dual-beam technique for deriving wind fields from airborne Doppler radar. Preprints, 25th Int. Conf. on Radar Meteorology, Paris, France, Amer. Meteor. Soc., 458-461.

Judt, F., and S. S. Chen, 2010: Convectively generated potential vorticity in rainbands and formation of the secondary eyewall in Hurricane Rita of 2005. J. Atmos. Sci., 67, 3581-3599, https://doi.org/10.1175/2010JAS3471.1.

Kepert, J. D., 2013: How does the boundary layer contribute to eyewall replacement cycles in axisymmetric tropical cyclones? J. Atmos. Sci., 70, 2808-2830, https://doi.org/10.1175/JAS-D-13-046.1.

Kimball, S. K., 2006: A modeling study of hurricane landfall in a dry environment. Mon. Wea. Rev., 134, 1901-1918, https://doi.org/ 10.1175/MWR3155.1.

Kossin, J. P., and M. Sitkowski, 2012: Predicting hurricane intensity and structure changes associated with eyewall replacement cycles. Wea. Forecasting, 27, 484-488, https://doi.org/10.1175/ WAF-D-11-00106.1.

—_, and M. DeMaria, 2016: Reducing operational hurricane intensity forecast errors during eyewall replacement cycles. Wea. Forecasting, 31, 601-608, https://doi.org/10.1175/ WAF-D-15-0123.1.

Lee, W.-C., B. Jou, P.-L. Chang, and S.-M. Deng, 1999: Tropical cyclone kinematic structure retrieved from single-Doppler radar observations. Part I: Interpretation of Doppler velocity patterns and the GBVTD technique. Mon. Wea. Rev., 127, 2419-2440, https://doi.org/10.1175/1520-0493(1999)127<2419: TCKSRF $>2.0 . C O ; 2$.

Maclay, K. S., M. DeMaria, and T. H. Vonder Haar, 2008: Tropical cyclone inner-core kinetic energy evolution. Mon. Wea. Rev., 136, 4882-4898, https://doi.org/10.1175/2008MWR2268.1.

Martinez, Y., G. Brunet, M. K. Yau, and X. Wang, 2011: On the dynamics of concentric eyewall genesis: Space-time empirical normal modes diagnosis. J. Atmos. Sci., 68, 457-476, https:// doi.org/10.1175/2010JAS3501.1.

May, P. T., and G. J. Holland, 1999: The role of potential vorticity generation in tropical cyclone rainbands. J. Atmos. Sci., 56, 1224-1228, https://doi.org/10.1175/1520-0469(1999)056<1224: TROPVG $>2.0 . \mathrm{CO} ; 2$.

Montgomery, M. T., and R. J. Kallenbach, 1997: A theory for vortex Rossby-waves and its application to spiral bands and intensity changes in hurricanes. Quart. J. Roy. Meteor. Soc., 123, 435-465, https://doi.org/10.1002/qj.49712353810.

_ J. A. Zhang, and R. K. Smith, 2014: An analysis of the observed low-level structure of rapidly intensifying and mature hurricane Earl (2010). Quart. J. Roy. Meteor. Soc., 140, 21322146, https://doi.org/10.1002/qj.2283.

Moon, Y., and D. S. Nolan, 2010: The dynamic response of the hurricane wind field to spiral rainband heating. J. Atmos. Sci., 67, 1779-1805, https://doi.org/10.1175/2010JAS3171.1.

Qiu, X., and Z.-M. Tan, 2013: The roles of asymmetric inflow forcing induced by outer rainbands in tropical cyclone secondary eyewall formation. J. Atmos. Sci., 70, 953-974, https:// doi.org/10.1175/JAS-D-12-084.1. 
,-- , and Q. Xiao, 2010: The roles of vortex Rossby waves in hurricane secondary eyewall formation. Mon. Wea. Rev., 138, 2092-2109, https://doi.org/10.1175/2010MWR3161.1.

Reasor, P. D., M. D. Eastin, and J. F. Gamache, 2009: Rapidly intensifying Hurricane Guillermo (1997). Part I: Low-wavenumber structure and evolution. Mon. Wea. Rev., 137, 603-631, https:// doi.org/10.1175/2008MWR2487.1.

Riemer, M., 2016: Meso- $\beta$-scale environment for the stationary band complex of vertically-sheared tropical cyclones. Quart. J. Roy. Meteor. Soc., 142, 2442-2451, https://doi.org/10.1002/qj.2837.

Rogers, R. F., S. Lorsolo, P. Reasor, J. Gamache, and F. Marks, 2012: Multiscale analysis of tropical cyclone kinematic structure from airborne Doppler radar composites. Mon. Wea. Rev., 140, 77-99, https://doi.org/10.1175/MWR-D-10-05075.1.

- , P. D. Reasor, and J. A. Zhang, 2015: Multiscale structure and evolution of Hurricane Earl (2010) during rapid intensification. Mon. Wea. Rev., 143, 536-562, https://doi.org/ 10.1175/MWR-D-14-00175.1.

Rozoff, C. M., D. S. Nolan, J. P. Kossin, F. Zhang, and J. Fang, 2012: The roles of an expanding wind field and inertial stability in tropical cyclone secondary eyewall formation. J. Atmos. Sci., 69, 2621-2643, https://doi.org/10.1175/JAS-D-11-0326.1.

Sawada, M., and T. Iwasaki, 2010: Impacts of evaporation from raindrops on tropical cyclones. Part I: Evolution and axisymmetric structure. J. Atmos. Sci., 67, 71-83, https://doi.org/ 10.1175/2009JAS3040.1.

Shapiro, L. J., and H. E. Willoughby, 1982: The response of balanced hurricanes to local sources of heat and momentum. J. Atmos. Sci., 39, 378-394, https://doi.org/10.1175/ 1520-0469(1982)039<0378:TROBHT>2.0.CO;2.

Sitkowski, M., J. P. Kossin, and C. M. Rozoff, 2011: Intensity and structure changes during hurricane eyewall replacement cycles. Mon. Wea. Rev., 139, 3829-3847, https://doi.org/10.1175/ MWR-D-11-00034.1.

_ $-\ldots$, and J. Knaff, 2012: Hurricane eyewall replacement cycle thermodynamics and the relict inner eyewall circulation. Mon. Wea. Rev., 140, 4035-4045, https://doi.org/ 10.1175/MWR-D-11-00349.1.

Smith, R. K., M. T. Montgomery, and S. V. Nguyen, 2009: Tropical cyclone spin-up revisited. Quart. J. Roy. Meteor. Soc., 135, 1321-1335, https://doi.org/10.1002/qj.428.

Stern, D. P., and F. Zhang, 2016: The warm-core structure of Hurricane Earl (2010). J. Atmos. Sci., 73, 3305-3328, https:// doi.org/10.1175/JAS-D-15-0328.1.

Stevenson, S. N., K. L. Corbosiero, and J. Molinari, 2014: The convective evolution and rapid intensification of Hurricane Earl (2010). Mon. Wea. Rev., 142, 4364-4380, https://doi.org/ 10.1175/MWR-D-14-00078.1.
Sun, Y. Q., Y. Jiang, B. Tan, and F. Zhang, 2013: The governing dynamics of the secondary eyewall formation of Typhoon Sinlaku (2008). J. Atmos. Sci., 70, 3818-3837, https://doi.org/ 10.1175/JAS-D-13-044.1.

Susca-Lopata, G., J. Zawislak, E. J. Zipser, and R. F. Rogers, 2015: The role of observed environmental conditions and precipitation evolution in the rapid intensification of Hurricane Earl (2010). Mon. Wea. Rev., 143, 2207-2223, https://doi.org/ 10.1175/MWR-D-14-00283.1.

Terwey, W. D., and M. T. Montgomery, 2008: Secondary eyewall formation in two idealized, full-physics modeled hurricanes. J. Geophys. Res., 113, D12112, https://doi.org/10.1029/ 2007JD008897.

Vigh, J. L., and Coauthors, 2016: FLIGHT+: The extended flight level dataset for tropical cyclones (version 1.1). Tropical Cyclone Data Project, National Center for Atmospheric Research, Research Applications Laboratory, Boulder, CO, accessed 16 May 2017, http://rpi.bios.edu/ publications/journals/flight-the-extended-flight-level-datasetfor-tropical-cyclones-version-11.

Willoughby, H. E., and M. B. Chelmow, 1982: Objective determination of hurricane tracks from aircraft observations. Mon. Wea. Rev., 110, 1298-1305, https://doi.org/10.1175/15200493(1982)110<1298:ODOHTF>2.0.CO;2.

_ J. A. Clos, and M. G. Shoreibah, 1982: Concentric eye walls, secondary wind maxima, and the evolution of the hurricane vortex. J. Atmos. Sci., 39, 395-411, https://doi.org/10.1175/ 1520-0469(1982)039<0395:CEWSWM>2.0.CO;2.

, F. D. Marks Jr., and R. J. Feinberg, 1984: Stationary and moving convective bands in hurricanes. J. Atmos. Sci., 41, 3189-3211, https://doi.org/10.1175/1520-0469(1984)041<3189: $\mathrm{SAMCBI}>2.0 . \mathrm{CO} ; 2$.

Wu, C.-C., Y.-H. Huang, and G.-Y. Lien, 2012: Concentric eyewall formation in Typhoon Sinlaku (2008). Part I: Assimilation of T-PARC data based on the ensemble Kalman filter (EnKF). Mon. Wea. Rev., 140, 506-527, https://doi.org/ 10.1175/MWR-D-11-00057.1.

Xu, J., and Y. Wang, 2010: Sensitivity of tropical cyclone inner-core size and intensity to the radial distribution of surface entropy flux. J. Atmos. Sci., 67, 1831-1852, https://doi.org/10.1175/2010JAS3387.1.

Yang, Y.-T., H.-C. Kuo, E. A. Hendricks, and M. S. Peng, 2013: Structural and intensity changes of concentric eyewall typhoons in the western north Pacific basin. Mon. Wea. Rev., 141, 2632-2648, https://doi.org/10.1175/MWR-D-12-00251.1.

Zhang, F., D. Tao, Y. Q. Sun, and J. D. Kepert, 2017: Dynamics and predictability of secondary eyewall formation in sheared tropical cyclones. J. Adv. Model. Earth Syst., 9, 89-112, https:// doi.org/10.1002/2016MS000729. 